\title{
Qualitative Vertiefungsanalyse
}

Die dritte Teilstudie der Arbeit ist eine qualitative Vertiefungsanalyse. In Kapitel 3 und Kapitel 4 wurde die Relevanz von sprachlichen und kontextuellen Veränderungen geschildert. Aus diesem Grund ist es das Ziel der qualitativen Vertiefungsanalyse, fachliche und kontextuelle Merkmale von Textaufgaben in Beziehung mit den in Kapitel 7 extrahierten sprachlichen Faktoren zu bringen. Dazu konnte die Faktorenwerte der Textaufgaben nach der Faktorenanalyse genutzt werden, um für die jeweiligen Faktoren charakteristische Aufgaben festzustellen. Diese ermittelten charakteristischen Aufgaben konnten durch ein deduktives - durch die in Abschnitt 3.4.3 beschriebenen Klassifizierungsmöglichkeiten von Aufgaben - und induktives - durch Sichtung der charakteristischen Textaufgaben (des Materials) - Vorgehen ausgewertet werden. Die Intention der Auswertung war die Bildung von Aufgabentypen, die mit den festgestellten sprachlichen Faktoren in einen Zusammenhang gebracht werden können.

Überblick: Für die qualitative Vertiefungsanalyse ergibt sich eine auf das Ziel ausgerichtete Methode zur Auswertung (Abschnitt 9.1). Die Auswertungsgrundlage für die qualitative Untersuchung ist nur ein Teil der Gesamtstichprobe an Textaufgaben, der durch ein in diesem Kapitel geschildertes Verfahren ausgewählt wurde (Abschnitt 9.1.1). Die Analyse der betrachteten Textaufgaben basiert auf einem Vorgehen aus der Literatur (Abschnitt 9.1.2). Zur Kodierung der Textaufgaben wurde ein Kodierschema mit sieben Hauptkategorien durch ein deduktiv-induktives Vorgehen entwickelt (Abschnitt 9.1.3). Um die qualitative Bildung der Hauptkategorien abzusichern, existieren Qualitätskriterien für das Kodierschema, die erfüllt werden müssen, damit das Kodierschema weiterverwendet werden kann (Abschnitt 9.1.4). Um die Spezifika der charakteristischen Aufgaben pro Faktor zu verdeutlichen, werden für die fünf Faktoren jeweils

D. Bednorz, Sprachliche Variationen von mathematischen Textaufgaben,

Bielefelder Schriften zur Didaktik der Mathematik 5,

https://doi.org/10.1007/978-3-658-33003-3_9 
zwei Textaufgaben exemplarisch dargestellt (Abschnitt 9.2). Dementsprechend wird für den erklärenden (Abschnitt 9.2.1), komprimierenden (Abschnitt 9.2.2), beschreibenden (Abschnitt 9.2.3), informativen (Abschnitt 9.2.4) und instruktiven (Abschnitt 9.2.5) Faktor beschrieben, welche Kategorien des Kodierschemas in den Textaufgaben für die jeweiligen Faktoren besonders bedeutsam sind. Die exemplarischen Darstellungen der besonders bedeutsamen Kategorien der jeweiligen Textaufgaben sollen zur Bildung von Aufgabentypen überleiten. Durch die Feststellung der Häufigkeit der Kodierung der Textaufgaben wird eine Interpretation der Aufgabentypen und damit die Deutung einer Bezeichnung erleichtert (Abschnitt 9.3). Zur Verdeutlichung der Bezeichnung der Aufgabentypen wird die Häufigkeit der Kodierung der Kategorien des Kodierschemas für den erklärenden (Abschnitt 9.3.1), komprimierenden (Abschnitt 9.3.2), beschreibenden (Abschnitt 9.3.3), informativen (Abschnitt 9.3.4) und instruktiven (Abschnitt 9.3.5) Faktor einzeln interpretiert. Die interpretierte Bezeichnung soll die Spezifika der Häufigkeit der Kodierung bündeln und wiedergeben. Nach der Darstellung der inhaltlichen und kontextuellen Spezifika der Textaufgaben je Faktor wird durch eine kontrastive Analyse zwischen den Textaufgaben der Faktoren eine Bezeichnung für einen Aufgabentyp abgeleitet (Abschnitt 9.3.6)

\subsection{Auswertungsmethode}

Überblick (Abschnitt 9.1): Das qualitative Vorgehen der dritten Teilstudie dieser Arbeit erforderte aufgrund der Zielsetzung eine passende Auswertungsmethode. Für die Analyse wurde ein Teil des vorhandenen Datensatzes verwendet. Entsprechend wird zunächst die Grundlage der Auswahl geschildert (Abschnitt 9.1.1). Die Untersuchung des Datensatzes orientierte sich maßgeblich an bereits etablierten Verfahren zur Analyse, die auf die vorliegende Analyse von Textaufgaben spezifiziert wurden (Abschnitt 9.1.2). Anhand von deduktiven und induktiven Erkenntnissen wurde ein Kodierschema zur Betrachtung der Textaufgaben konzipiert (Abschnitt 9.1.3). Die Qualität des Kodierschemas wurde anhand den in der Literatur erörterten Kriterien eingeschätzt (Abschnitt 9.1.4). 


\subsubsection{Auswertungsgrundlage}

Basis für die qualitative Vertiefungsanalyse ist die in Kapitel 7 durchgeführte explorative Faktorenanalyse der quantitativ erhobenen Textmerkmale. Für die Vertiefungsanalyse ist es notwendig, Textaufgaben zu betrachten, die jeweils nur für einen Faktor besonders repräsentativ sind - gekennzeichnet durch die Textmerkmale, die in der Textaufgabe vorkommen und eine hohe Ladung auf den Faktor aufweisen (vgl. Kapitel 7). Das bedeutet, wenn eine Textaufgabe viele Textmerkmale aufweist, die sowohl auf den ersten als auch auf den zweiten Faktor hoch laden, lässt sich nicht sagen, ob die kontextuellen und fachlichen Besonderheiten für die Textaufgaben des ersten oder zweiten Faktors typisch sind. Durch die Ermittlung der Textmerkmale für die in der Arbeit genutzten Textaufgaben konnte für jede Textaufgabe ein Faktorenwert berechnet werden. Die Faktorenwerte wurden infolge der Faktorenextraktion z-standardisiert, was bedeutet, dass die Faktorenwerte auf 0 normiert wurden. Für z-standardisierte Faktorenwerte bedeutet eine Standardabweichung größer gleich \pm 1 , dass sich die Werte bedeutsam vom Mittel unterscheiden und den jeweiligen Faktor besonders stark repräsentieren. Das Ziel war es daher, Textaufgaben für die qualitative Vertiefungsstudie zu selektieren, die eine Standardabweichung größer 1 auf einen Faktor besitzen und gleichzeitig - da die Textaufgaben sich nur bedeutsam für einen Faktor vom Mittel unterscheiden sollen - eine Standardabweichung von kleiner 1 für die restlichen vier Faktoren aufweisen. In Abbildung 9.1 sind die Faktorenwerte je Faktor abgetragen. In der Abbildung ist über eine horizontale Gerade eine Standardabweichung markiert.

Im Hinblick auf das Ziel, Textaufgaben auszuwählen, die eine Standardabweichung größer 1 auf einen Faktor und auf den restlichen Faktoren eine Standardabweichung kleiner 1 haben, wurden die Textaufgaben, deren Faktorenwerte über der abgebildeten Geraden liegen, gefiltert. Durch die geschilderte Auswahl der Textaufgaben anhand der Faktorenwerte wurden aus dem Gesamtdatensatz an Textaufgaben von $N=348$ für die qualitative Vertiefungsstudie $N=$ 49 Textaufgaben ausgewählt. Für den ersten, zweiten, dritten und fünften Faktor wurden $N=10$ Textaufgaben und für den vierten Faktor $N=9$ Textaufgaben selektiert.

Anschließend soll beschrieben werden, wie die Analyse der $N=49$ ausgewählten Textaufgaben erfolgt ist. 


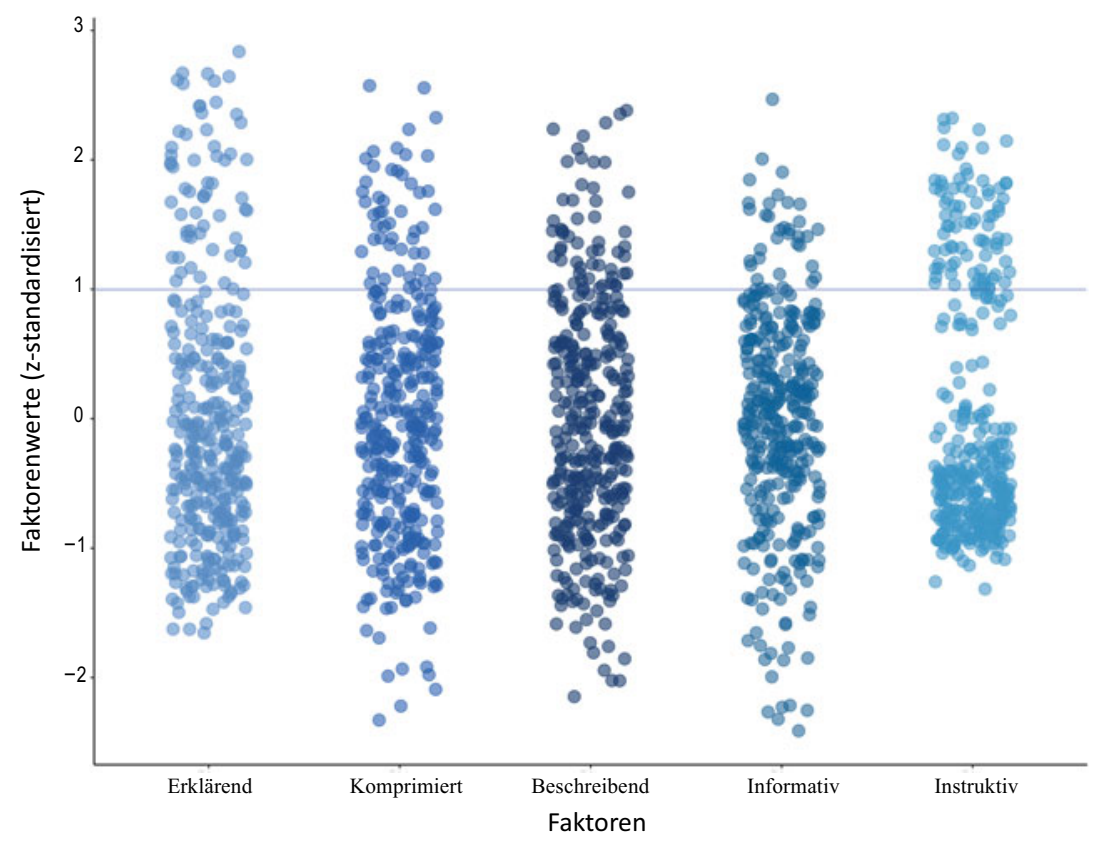

Abbildung 9.1 Darstellungen der Faktorenwerte der Textaufgaben für jeden Faktor und Markierung einer Standardabweichung (Eigene Erstellung)

\subsubsection{Ablaufmodell der Analyse}

Die Vertiefungsanalyse mit den $N=49$ Textaufgaben orientiert sich an der qualitativen Inhaltsanalyse nach Mayring $(2015,2016)$. Letztere eignet sich in diesem Rahmen, da für die ausgewählten Textaufgaben der jeweiligen Faktoren keine vorhandenen Kenntnisse über bestimmte kontextuelle und fachliche Eigenschaften bestehen. Demnach muss für die Analyse der kontextuellen und fachlichen Eigenschaften der Textaufgaben explorativ vorgegangen werden. Die qualitative Inhaltsanalyse eignet sich zur Untersuchung von schriftlichen Texten und hat eine explorative Schwerpunktsetzung, die für den Rahmen der Arbeit genutzt wurde, um die Textaufgaben zu analysieren. 
Die qualitative Auswertung richtete sich nach dem Vorgehen von Mayring (2016). Das Vorgehen der qualitativen Inhaltsanalyse wurde für die eigenen Forschungszwecke an das Studiendesign und die Analyse von Textaufgaben adaptiert. Es kann in fünf Schritte unterteilt werden:

1. Sichtung: Die $N=49$ Textaufgaben wurden zunächst betrachtet und auffallende kontextuelle und inhaltliche Charakteristika jeder Aufgabe wurden notiert.

2. Deduktion: Die kontextuellen und inhaltlichen Charakteristika wurden mit bereits etablierten Klassifikationen von Mathematikaufgaben verknüpft, die in Abschnitt 3.4.3 dargestellt wurden. Insbesondere erfolgte eine Orientierung an dem Klassifikationsschema von Jordan et al. (2006). Dadurch entstanden die ersten Hauptkategorien, deren Subkategorien aus der Literatur übernommen und für den Datensatz der Textaufgaben in dieser Arbeit angepasst wurden. Durch die deduktive Betrachtung wurden folgende fünf Hauptkategorien ausgewählt: mathematisches Argumentieren, Gebrauch von mathematischen Darstellungen, mathematische Tätigkeit, Daten und Informationen, Transferbezug.

3. Induktion: In einer zweiten Sichtung wurden die $N=49$ Textaufgaben ein weiteres Mal analysiert, um zusätzliche Charakteristika zu selektieren, die nicht von den bereits etablierten Klassifikationsmöglichkeiten abgedeckt werden. Dadurch konnte die Spezifität der ausgewählten Textaufgaben abgebildet werden. Dies betraf besonders kontextuelle Aspekt der Aufgabengestaltung. Die zweite Sichtung führte zur Ergänzung von zwei Hauptkategorien: Interaktion und strukturelle Gestalt.

Es ist zu erkennen, dass durch die Hauptkategorien sowohl inhaltliche (mathematische) Kriterien (mathematisches Argumentieren, Gebrauch von mathematischen Darstellungen, mathematische Tätigkeit, Daten und Informationen) als auch weitere allgemeine kontextuelle Kriterien (Transferbezug, Interaktion, strukturelle Gestalt) betrachtet werden.

4. Kodierschema und Prüfung: Aus den sieben Hauptkategorien ergibt sich ein Kodierschema, mit dem die Textaufgaben kodiert werden konnten. Um die Qualität der Kodierung zu überprüfen, wurde als Gütemaß Krippendorffs $\alpha$ berechnet.

5. Bildung von Aufgabentypen: Durch die Kodierung der Textaufgaben ergaben sich für die einzelnen Textaufgaben für die Faktoren Kodierungstendenzen. Die 
Kodierungstendenz ist durch die Häufigkeit der Kodierungen der Subkategorien je Hauptkategorien für einen Faktor bestimmt. Diese Häufigkeit je Faktor kann als Maß für die inhaltlichen und kontextuellen Besonderheiten der Textaufgaben interpretiert werden. Aus den unterschiedlichen Ausprägungen wurde eine Bezeichnung für die Textaufgaben je Faktor gewählt.

Nachfolgend sollen die Hauptkategorien und das Kodierschema näher beschrieben werden.

\subsubsection{Hauptkategorien und Kodierschema}

Wie in Abschnitt 9.1.2 geschildert wurde, wurden durch die ersten drei Schritte in einem deduktiv-induktiven Ansatz sieben Hauptkategorien gebildet. Die deduktive Kategorienbildung bezieht sich insbesondere auf das in Abschnitt 3.4.3 erläuterte Klassifikationsschema von Jordan et al. (2006) und wurde entsprechend an die vorhandenen $N=49$ Textaufgaben angepasst und reduziert. In Abbildung 9.2 sind im oberen Bereich die fünf Hauptkategorien (mathematisches Argumentieren, Gebrauch von (mathematischen) Darstellungen, mathematische Tätigkeit, Daten und Informationen, Transferbezug) abgebildet, die durch das deduktive Verfahren ermittelt worden sind. Die Subkategorien wurden ebenfalls von Jordan et al. (2006) übernommen und für den vorliegenden Datensatz (Textaufgaben) angepasst. Sie sind in Abbildung 9.2 den jeweiligen Hauptkategorien zugeordnet. Im dritten Punkt in Abschnitt 9.1.2 wurde erläutertet, dass durch ein induktives Vorgehen weitere Hauptkategorien bestimmt worden sind. Die beiden induktiv ermittelten Hauptkategorien (Interaktion und strukturelle Gestalt) sind im unteren Bereich der Abbildung 9.2 dargestellt. Durch die Sichtung der Textaufgaben wurden unterschiedliche Subkategorien der induktiv ermittelten Hauptkategorien differenziert. Die Subkategorien sind den jeweiligen beiden unteren induktiv ermittelten Hauptkategorien in Abbildung 9.2 zugeordnet.

Um die einzelnen Hauptkategorien näher zu beschreiben und zu verdeutlichen, aus welchem Grund die gewählte Differenzierung in Subkategorien gewählt wurde, wird im Folgenden näher auf die Kategorien eingegangen und die inhaltlichen und kontextuellen Kriterien, die für die Kodierung verwendet worden sind, werden erläutert.

Mathematisches Argumentieren: Das mathematische Argumentieren beschreibt die Art der Anforderungen an das Lösungsverhalten der Lernenden. Im einfachsten Fall werden in der Aufgabe rechnerische Argumente verlangt. Bei einer 


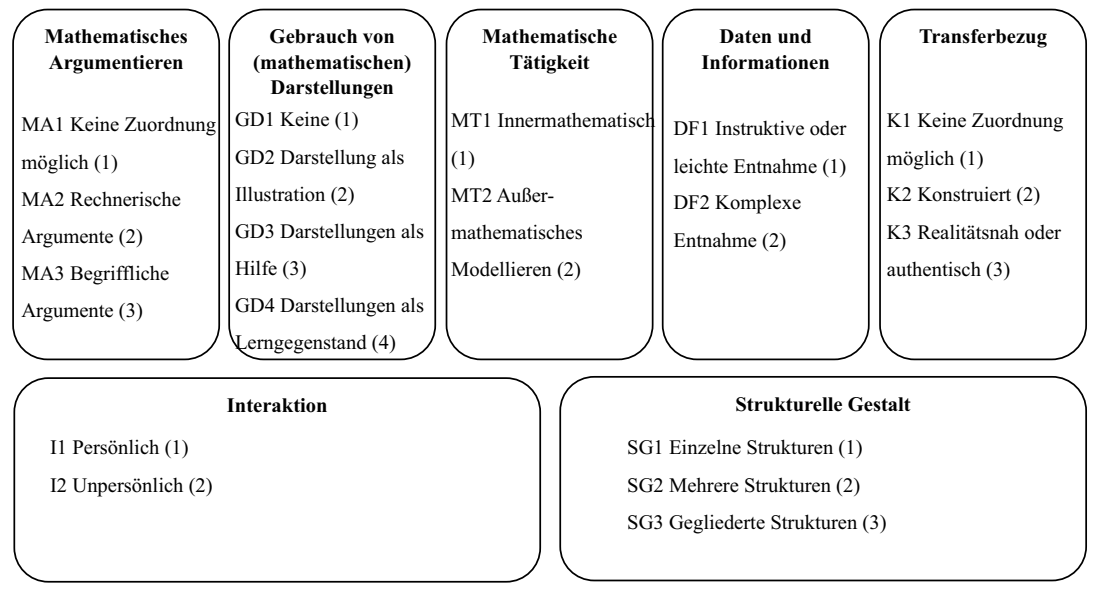

Abbildung 9.2 Kategoriensystem mit inhaltlichen und kontextuellen Kriterien für Textaufgaben (Eigene Erstellung)

solchen Aufgabe ist es ausreichend, Zahlenwerte zur Aufgabenlösung anzugeben. Typische Operatoren für eine solche Aufgabe sind: Rechne, Gib an, angeben etc. Bei Aufgaben, die begriffliche Argumente erfordern, ist eine Lösung durch die Verwendung und Darlegung von mathematischen Begrifflichkeiten nötig. Dies kann durch eine reflexive Auseinandersetzung mit der Aufgabe erfolgen, indem beispielsweise Daten und Informationen verglichen oder Begriffe unter den Aspekten von Beweisführungen und Argumentationsketten verwendet werden müssen.

Gebrauch von (mathematischen) Darstellungen: Der Gebrauch von (mathematischen) Darstellungen ist ein zentraler Aspekt von mathematischen Handlungen. Rechnerische Aufgaben, beispielsweise Übungsaufgaben, haben häufig keine Darstellungsformen, die die Textaufgabe unterstützen. Bei anderen Aufgaben können Darstellungen als Illustration dienen. Sie fungieren dabei nicht als Träger von Informationen, sondern dienen lediglich als Unterstützung dazu, die Situation im Text genauer zu verstehen. So können illustrative Darstellungen dazu verwendet werden, Informationen bei einer Modellierung abzuschätzen. Daneben können Darstellungen als Hilfsstrukturen genutzt werden - beispielsweise, indem bereits das mathematische Modell von der vorliegenden Situation skizziert ist. Außerdem können (mathematische) Darstellungen als Tabellen oder Diagramme, besonders für die Informations- und Datenentnahme in Mathematikaufgaben, vorkommen. Da Darstellungen auch mathematische Objekte abbilden können, dienen 
sie schließlich selbst auch als zentraler Lerngegenstand. Hierbei sind die Stoffgebiete der Geometrie und Stochastik zentrale inhaltliche Felder, an denen mit Darstellungen operiert, konstruiert und bewiesen wird.

Mathematische Tätigkeit: Für die Mathematik lassen sich zwei grundlegende mathematische Tätigkeiten unterscheiden: zum einen innermathematische Aufgaben, also solche, die sich bei der Unterteilung zwischen Realität und Mathematik ausschließlich auf die abstrakte Welt der Mathematik beziehen, zum anderen die außermathematischen Modellierungsaufgaben. Für letztere Aufgaben ist eine Übersetzungsleistung in die Realität vorzunehmen; es ist dementsprechend außermathematisch zu modellieren. Eine außermathematische Modellierung erfordert dabei viele Teilkompetenzen, die durchlaufen werden müssen und in Abschnitt 3.2.5 diskutiert wurden.

Daten und Informationen: Die Entnahme der Daten und Informationen aus einer Textaufgabe ist ein zentraler Aspekt zur Lösung einer Aufgabe. Je unklarer und schwieriger die Entnahme von bedeutenden Informationen ist, desto schwieriger ist die Lösung der Aufgabe. Die Daten und Informationen können innerhalb einer Instruktion eingebettet sein, in der das Datenmaterial häufig als Zahl vorliegt. Außerdem können sie aus Tabellen entnommen werden, was einer offensichtlichen Entnahme der Daten und Informationen entspricht. Daneben können sie über eine lineare Entnahme gewonnen werden, die (zumindest teilweise) der Lösungsstruktur entspricht. Darüber hinaus sind weitere, komplexere Entnahmen von Daten und Informationen denkbar, beispielsweise eine nicht lineare Entnahme oder fehlende klare Bezüge zu Daten und Informationen, die für die Lösung verwendet werden können.

Transferbezug: In Verbindung mit der mathematischen Tätigkeit steht der Transferbezug. Aufgaben können dabei konstruiert sein, wenn eine reale Situation um einen mathematischen Gegenstand gerahmt wird. Die Problematisierung ist in einem solchem Fall häufig erdacht und besitzt keine außerschulische Relevanz. Im Gegensatz dazu besitzen realitätsnahe und authentische Aufgaben eine Problematisierung, die an reale Situationen (mindestens) angelehnt ist. Eine solche Problematisierung besitzt jedoch nicht zwingend auch eine außerschulische Relevanz.

Interaktion: Die Kategorie Interaktion bildet ab, dass Aufgaben verschiedene interpersonale Ebenen einnehmen können. Dominant sind hierbei zum einen die Ausprägung von persönlichen bzw. narrativen Ebenen und zum anderen die unpersönlichen Ebene der textuellen Formulierung. Die persönlichen bzw. narrativen 
Ebenen zeigen sich bei Aufgaben, indem die Namen von Akteurinnen und Akteuren, Personalpronomen sowie weitere sprachliche Mittel zur Personalisierung des Kontextes verwendet werden. Eine unpersönliche Ebene tritt auf, wenn passive Strukturen genutzt werden und es vermieden wird, Akteurinnen und Akteure sowie Personen zu benennen. Eine Objektivierung der Situation steht im Vordergrund und handelnde Subjekte werden nicht betrachtet. Mit der Hauptkategorie Interaktion ist die Ebene des im Aufgabenkontext vermittelten Inhaltes gemeint und nicht etwaige Aufgabenimperative, die meistens einen direkten Bezug zur Rezipientin oder zum Rezipienten aufweisen. Dahingehend wurden für die Kodierung der Interaktion die Instruktionen in der Aufgabenstellung der Textaufgaben nicht betrachtet.

Strukturelle Gestalt: Wird die Aufgabe im Gesamten betrachtet, lassen sich Unterschiede in der strukturellen Gestalt von Aufgaben erkennen. Eine Form sind einzelne Strukturen, in die sich eine einzelne Aufgabenstellung bzw. ein Aufgabenabsatz gliedert. Eine andere Variante sind mehrere Strukturen, die sich durch mehrere Teilaufgaben auszeichnen. Häufig kommt es zu einer Trennung von Kontext und Aufgabenstellung. Eine komplexere strukturelle Aufteilung zeigen gegliederte Strukturen. Diese Aufgaben sind aufbauend strukturiert und haben mehr als zwei Teilaufgaben und mehrere Aufgabenabsätze. Die Struktur kann sich differenzieren in Kontext, Daten, Darstellungen und Aufgabenstellung mit verschiedenen Anordnungsvarianten.

Um einzuschätzen, inwieweit sich das Kodierschema zur Kodierung der Textaufgaben eignet, werden nachfolgend Qualitätskriterien geprüft.

\subsubsection{Qualitätskriterien des Kodierschemas}

Die Herausforderung von qualitativen Studien ist die Einhaltung der Ansprüche einer empirischen Arbeit. Dahingehend ist es für den qualitativen Forschungsteil dieser Studie zentral, Gütekriterien zu prüfen und einzuhalten. Zur Prüfung der Güte der qualitativen Studie wurden die Gütekriterien nach Krippendorff (2009) verwendet.

Es ergeben sich acht inhaltsanalytische Gütekriterien, die die Qualität der qualitativen Inhaltsanalyse für eine empirische Arbeit beschreiben (Krippendorff, 2009; Mayring, 2015). Diese acht Kriterien sind: semantische Gültigkeit, Stichprobengültigkeit, korrelative Gültigkeit, Vorhersagegültigkeit, Konstruktgültigkeit, Stabilität, Reproduzierbarkeit und Exaktheit. Aufgrund des explorativen 
Verfahrens der Analyse werden die Kategorien Korrelative Gültigkeit und Vorhersagegültigkeit nicht betrachtet.

Semantische Validität: Die semantische Validität dient zur Prüfung insbesondere der Definition der Kategorien. Dies umfasst die Prüfung der Definitionen, der Auswahl der Ankerbeispiele und der Kodierregeln. Geprüft werden kann die semantische Validität beispielsweise durch Expertenbeurteilungen. Dahingehend wurden die verwendeten Definitionen und die Kodierregeln mit Fachleuten diskutiert und iterativ modifiziert. Die Auswahl der Ankerbeispiele zur Kodierung erfolgte anhand des in Abschnitt 9.1.2 beschriebenen Vorgehens. Durch die Auswahl von charakteristischen Aufgaben je Faktor können die Ankerbeispiele als repräsentativ betrachtet werden. Aufgrund der Beurteilung und Diskussion von Expertinnen und Experten sowie der Verbesserung der Definitionen und Kodierregeln und durch die systematische und quantifizierte Auswahl der Ankerbeispiele kann von einer hohen semantischen Validität ausgegangen werden.

Stichprobengültigkeit: Die Stichprobengültigkeit bezieht sich auf die Ziehung von systematischen Stichproben aus der Auswahl - im vorliegenden Fall von Textaufgaben. Der Datensatz, der sich aus der systematischen Stichprobenziehung ergibt, soll die Repräsentativität für alle anderen Aufgaben gewährleisten. In der Studie umfasst die Stichprobenziehung die Auswahl der Textaufgaben aus den Schulbüchern. Die Stichprobenauswahl erfolgte wie in Abschnitt 6.5 beschrieben durch das systematische Ziehen von Textaufgaben aus unterschiedlichen Schulbüchern. Eingeschränkt ist die Repräsentativität in der Weise, dass die Schulbücher aus dem Bundesland Nordrhein-Westfalen stammen. Entsprechend kann von einer validen Stichprobengültigkeit ausgegangen werden, unter der Limitierung der Auswahl der Textaufgaben aufgrund des föderalistischen Systems in der Bundesrepublik.

Konstruktgültigkeit: Anhand der Konstruktgültigkeit wird überprüft, inwieweit die verwendeten Methoden die erfassten Merkmale tatsächlich messen. Die explorative und die konfirmatorische Faktorenanalyse sind Verfahren, um die Konstruktgültigkeit zu prüfen. Da vor der qualitativen Vertiefungsanalyse eine explorative Faktorenanalyse durchgeführt wurde, kann von einer Konstruktgültigkeit ausgegangen werden.

Reproduzierbarkeit: Zur Prüfung der Reproduzierbarkeit wurde die Interraterreliabilität berechnet. Hierfür wurden durch eine Kodierschulung zwei Rater geschult. Anschließend kodierten diese die $N=49$ Aufgaben nach dem in Abbildung 9.2 dargestellten Kategoriensystem. 
Die Ergebnisse der Interraterreliabilität sind in Tabelle 9.1 abgebildet. Alle Hauptkategorien erreichen einen Wert von Krippendorffs $\alpha>0.7$, der als Schwellenwert für die Güte der Reliabilität gilt (Hayes \& Krippendorff, 2007; Krippendorff, 2004, 2009). Damit weist das Kategoriensystem eine zufriedenstellende Reproduzierbarkeit auf. Die Hauptkategorien Mathematisches Argumentieren, Gebrauch von (mathematischen) Darstellungen, Daten und Informationen sowie strukturelle Gestalt erreichen einen Krippendorffs $\alpha>0.8$. Den höchsten Wert von Krippendorffs $\alpha$ weist die Hauptkategorie Daten und Informationen auf.

Damit erreicht das Kategoriensystem insgesamt ein gutes Ergebnis für die Reproduzierbarkeit der Kodierung der Hauptkategorien.

Tabelle 9.1 Interraterreliabilität der Kodierung (Reproduzierbarkeit)

\begin{tabular}{l|l|l|l|l}
\hline Kategorie & $N$ & Krippendorffs $\alpha$ & $95-\%-$ KI & Interpretation \\
\hline Mathematisches Argumentieren & 49 & 0.85 & $0.71-0.96$ & Gut \\
\hline $\begin{array}{l}\text { Gebrauch von (mathematischen) } \\
\text { Darstellungen }\end{array}$ & 49 & 0.89 & $0.75-1.00$ & Gut \\
\hline Mathematische Tätigkeit & 49 & 0.73 & $0.52-0.91$ & Akzeptabel \\
\hline Daten und Informationen & 49 & 0.91 & $0.74-1.00$ & Gut \\
\hline Transferbezug & 49 & 0.70 & $0.50-0.87$ & Akzeptabel \\
\hline Interaktion & 49 & 0.78 & $0.56-0.96$ & Akzeptabel \\
\hline Strukturelle Gestalt & 49 & 0.86 & $0.71-0.96$ & Gut \\
\hline
\end{tabular}

Stabilität: Zur Prüfung der Stabilität der Reproduzierbarkeit wurde die Intraraterreliabilität berechnet. Dies erfolgte, indem die $N=49$ Textaufgaben durch denselben Rater nach eineinhalb Monaten ein weiteres Mal kodiert wurden.

Die Ergebnisse der Intraraterreliabilität sind in Tabelle 9.2 dargestellt. Alle Hauptkategorien erreichen hierbei ein Krippendorffs $\alpha>0.7$. Damit wird der Schwellenwert für die Reliabilität erreicht und die Hauptkategorien weisen eine zufriedenstellende Stabilität auf. Insgesamt erreichen 5 Hauptkategorien einen Krippendorffs $\alpha>0.8$. Den höchsten Wert weisen die Hauptkategorien Gebrauch von (mathematischen) Darstellungen und Interaktion auf, die damit als besonders stabil erachtet werden können.

Exaktheit: Die Exaktheit setzt die Kriterien der Reproduzierbarkeit und der Stabilität des Kategoriensystems voraus. Wie bereits beschrieben, erreichen die Kriterien der Reproduzierbarkeit und Stabilität für das Kategoriensystem zufriedenstellende Ergebnisse. Zur Entwicklung der Exaktheit wurden Unstimmigkeiten 
Tabelle 9.2 Intraraterreliabilität der Kodierung (Stabilität der Reproduzierbarkeit)

\begin{tabular}{l|l|l|l|l}
\hline Kategorie & $N$ & Krippendorffs $\alpha$ & $95-\%-$ KI & Interpretation \\
\hline Mathematisches Argumentieren & 49 & 0.81 & $0.62-0.96$ & Gut \\
\hline $\begin{array}{l}\text { Gebrauch von (mathematischen) } \\
\text { Darstellungen }\end{array}$ & 49 & 0.87 & $0.73-0.97$ & Gut \\
\hline Mathematische Tätigkeit & 49 & 0.82 & $0.63-0.96$ & Gut \\
\hline Daten und Informationen & 49 & 0.78 & $0.45-1.00$ & Akzeptabel \\
\hline Transferbezug & 49 & 0.73 & $0.50-0.87$ & Akzeptabel \\
\hline Interaktion & 49 & 0.87 & $0.56-0.96$ & Gut \\
\hline Strukturelle Gestalt & 49 & 0.86 & $0.71-0.96$ & Gut \\
\hline
\end{tabular}

im Kategoriensystem geklärt und Begriffliches wurde eindeutiger beschrieben. Einzelne Unterkategorien, die nicht eindeutig unterscheidbar waren, wurden zusammengelegt, um so ein zwar gröberes, aber exakteres Kategoriensystem zu erhalten.

\subsection{Fallanalyse spezifischer Aufgaben aus den Faktoren}

Um die Spezifika der ausgewählten charakteristischen Aufgaben für die unterschiedlichen Faktoren zu verdeutlichen, werden in diesem Kapitel jeweils zwei Aufgaben präsentiert. Das Ziel der Fallanalyse ist es, exemplarisch aufzuzeigen, welche der in Abschnitt 9.1 erörterten Haupt- und Subkategorien für den jeweiligen Faktor besonders relevant sind.

Überblick (Abschnitt 9.2): Zur Verdeutlichung der relevanten Haupt- und Subkategorien werden nachfolgend für den erklärenden (Abschnitt 9.2.1), komprimierenden (Abschnitt 9.2.2), beschreibenden (Abschnitt 9.2.3), informativen (Abschnitt 9.2.4) und instruktiven (Abschnitt 9.2.5) Faktor kapitelweise zwei charakteristische Aufgaben beschrieben.

\subsubsection{Faktor: erklärend}

Die erste charakteristische Textaufgabe des erklärenden Faktors stammt aus der sechsten Klasse. In der Textaufgabe sollen Brüche miteinander verglichen und die Brüche geordnet werden.

Aufgabe 1 - Faktor: erklärend 
Jannik will einige Brüche vergleichen. Bei $\frac{1}{4}$ und $\frac{3}{4}$ sieht er sofort, welcher Bruch größer ist. In schwierigeren Fällen hilft ihm eine Zeichnung.

a) Vergleiche die Brüche durch Hinsehen oder durch Einteilen und Färben in einem Rechteckmodell. Setze das entsprechende Zeichen $<$, > oder $=$ ein.

$$
\frac{1}{4}-\frac{1}{3} \quad \frac{4}{6}-\frac{2}{3} \quad \frac{10}{12}-\frac{3}{4} \quad \frac{7}{12}-\frac{1}{2} \quad \frac{1}{6}-\frac{2}{12}
$$

b) Ordne alle Brüche aus der Teilaufgabe a) der Größe nach. Beschreibe, wie du vor gehst und formuliere Regeln (Körner et al., 2013, S. 92).

Die Textaufgabe zeichnet sich durch begriffliche Argumente aus. So ist es für die erste Teilaufgabe bedeutsam, die Brüche miteinander zu vergleichen und in der zweiten Teilaufgabe die gewählte Ordnung der Zahlen zu begründen. Hierbei ist es zwingend erforderlich, begriffliche Argumente zu verwenden. Auch die Formulierung als Regel kann als begrifflich dahingehend verstanden werden, dass die in der ersten Teilaufgabe durchgeführte Ordnung in eine Regel verallgemeinert werden soll. Die zweite Teilaufgabe verlangt begriffliche Argumente in einer reflexiven Weise.

In der Aufgabe werden mathematischen Darstellungen verwendet (nicht abgebildet). Die Darstellung dient dabei als Hilfe, um die numerischen Notationen miteinander zu vergleichen und daraus Regeln abzuleiten.

Die erste Aufgabe des erklärenden Faktors ist eine solche im innermathematischen Tätigkeitsbereich aus dem Gebiet der Algebra, ohne Zuordnungsmöglichkeiten zu einem bestimmten Transferbezug.

Die Daten und Informationen sind problemlos zu entnehmen. Die zu sortierenden relevanten Zahlen sind direkt sichtbar und wurden durch die Formatierung der Aufgabe von den anderen Textteilen abgehoben.

In der Aufgabe wird persönlich interagiert. Damit kennzeichnet die Aufgabe eine aktive Einbindung der Rezipientinnen und Rezipienten. Im ersten Teil wird von Jannik, er und ihm gesprochen, während in den anschließenden Fragestellungen die Rezipientin oder der Rezipient direkt angesprochen wird (Du).

Ein weiteres Merkmal dieser Aufgabe ist die aufbauende Struktur. Die gegliederte Struktur zeichnet sich dadurch aus, dass im ersten Teil der Aufgabe die Aufgabenstellung kontextualisiert wird. Nachfolgend sind die Daten der Aufgabe und die Arbeitsaufträge, voneinander abgesetzt. Dadurch entstehen Textebenen, die unterschiedliche Funktionen in der Aufgabe erfüllen. 
Die zweite charakteristische Aufgabe des erklärenden Faktors ist eine Textaufgabe aus der fünften Klasse. Es handelt sich um eine Aufgabe, in der Flächen miteinander verglichen und gewisse Strategien des Vergleichens evaluiert werden sollen.

Die zweite charakteristische Textaufgabe lässt sich keiner Subkategorie des mathematischen Argumentierens zuordnen, da in dieser Aufgabe nur ein Vergleich der Flächen stattfindet soll. Es ist nicht beschrieben, ob eine Erklärung des gewählten Zugangs zum Flächenvergleich erfolgen soll.

Aufgabe 2 - erklärend

Flächen nach ihrer Größe vergleichen! Pia, Merve und Ole haben sich jeweils einen Ansatz überlegt, wie man zwei Flächen nach ihrer Größe vergleichen kann. ,Ich lege die Fläche aufeinander', ,Ich lege beide Flächen mit vielen kleinen gleichen Flächen aus. Die zähle ich ab“. ,Ich zerschneide eine Fläche und lege sie neu zusammen, sodass ich sie besser vergleichen kann. ‘

a.) Wähle aus dem Arbeitsblatt Flächenvergleich zwei Flächen aus und vergleiche die beiden Flächen mit einem der Ansätze. Überlege zuerst, welcher Ansatz für die ausgesuchten Flächen geeignet ist.

b.) Wähle zwei weitere Flächen zum Vergleichen. Nutze diesmal einen der anderen Ansätze, um sie zu vergleichen. Vergleiche verschiedene Flächen so oft, bis du jeden Ansatz ausprobiert hast (Prediger et al., 2014, S. 176).

Für die Aufgabe ist der Gebrauch von mathematischen Darstellungen notwendig. Es sollen aus einem Arbeitsblatt unterschiedliche Flächen verwendet werden. Die Darstellungen können in diesem Fall als Darstellungen als Lerngegenstand betrachtet werden, da der Flächenvergleich als Hinführung zur Berechnung des Flächeninhaltes dient.

Die Aufgabe ist dem innermathematischen Bereich der Geometrie zuzuordnen und hat dementsprechend keinen Transferbezug.

Die Daten und Informationen sind in der Aufgabe problemlos ablesbar. Die Informationen zum Vergleich der Flächen werden in dem ersten Abschnitt gegeben. Dabei wird deutlich, dass insgesamt drei unterschiedliche Verfahren durch die Figuren der Aufgabe präsentiert werden. Die Auswahl der Flächen gelingt ebenfalls problemlos durch die Auswahl am Arbeitsblatt.

Aufgrund der Figuren als Protagonistinnen und Protagonisten findet im ersten Teil der Aufgabe eine persönliche Interaktion statt, die über einen Dialog der unterschiedlichen Ansätze erfolgt.

Die zweite charakteristische Aufgabe ist an der gegliederten Struktur erkennbar. So wird in der Beschreibung zwischen der Situation und den beiden 
Aufgabenstellungen unterschieden. Die Beschreibung der Situation stellt die kontextuelle Rahmung der Gegebenheit dar, die einen nahen Bezug zur Realität der Lernenden in einem schulischen Kontext aufweist. Die Aufgabenstellungen sind relativ separiert von der kontextuellen Rahmung (direkter Verweis auf das Arbeitsblatt) und beschreiben Aufgabenanforderungen, die mit den diskutierten Verfahren in der kontextuellen Rahmung zu tun haben.

Für die beiden exemplarisch dargestellten Beispiele für charakteristische Aufgaben aus dem erklärenden Faktor deutet sich an, dass begriffliche Argumente, eine aktive Einbindung der Rezipientin bzw. des Rezipienten, innermathematische Aufgaben, eine relativ problemlose Entnahme der Informationen und Daten sowie die strukturelle Gestalt des Aufbaus der Textaufgabe bedeutsame Kategorien dieser Gruppe an Textaufgaben sind.

\subsubsection{Faktor: komprimierend}

Die erste charakteristische Textaufgabe des komprimierenden Faktors ist eine Aufgabe für die fünfte Klasse aus dem Inhaltsbereich Geometrie. In der Textaufgabe sollen die Eigenschaften von Diagonalen in unterschiedlichen Vierecken beschrieben werden. Anhand der Aufgabenstellung zeichnet sich die Textaufgabe durch begriffliche Argumente aus, die aus der Konstruktion der Diagonalen in Vierecken folgen.

Die genutzten mathematischen Darstellungen (nicht abgebildet) in dieser Aufgabe (in der Abbildung ist ein Rechteck mit den Diagonalen AD und BC abgetragen) sind nicht nur eine Hilfe. Die Diagonalen und die Vierecke sind selbst Gegenstand des Lernens, durch die bedeutende Symmetrieeigenschaften von Vierecken entdeckt und begrifflich gefestigt werden sollen.

Aufgabe 1 - Faktor: komprimierend

Die rot eingezeichneten Strecken im Viereck heißen Diagonalen. Zeichne besondere Vierecke mit ihren Diagonalen. Untersuche ihre Eigenschaften.

- In welchen Vierecken sind die Diagonalen gleich lang?

- In welchen Vierecken stehen die Diagonalen zueinander senkrecht?

- In welchen Vierecken halbieren sich die Diagonalen? (Göckel et al., 2014, S. 110).

Bei der Aufgabe handelt es sich um eine innermathematische Aufgabe aus dem Bereich der Geometrie, die keinen Transferbezug aufweist. 
Die Informationen für diese Aufgabe sind durch die genutzte Abbildung problemlos zu entnehmen und können für die Fragestellung als Beispiel genutzt und auf weitere Vierecke übertragen werden.

Bezüglich der Interaktion zeigt die Aufgabe eine generell unpersönliche Interaktion. Der zweite Satz der Aufgabe ist als Imperativ formuliert und weist die Rezipientin oder den Rezipienten darauf hin, was in der Aufgabe verlangt wird.

Daneben werden in der Aufgabe mehrere Strukturen verwendet, die in einem Textkern gemeinsam vorkommen. Die Strukturen zeigen sich in der Gliederung der Aufgabenstellung mit einer zusätzlichen Darstellung.

Die zweite charakteristische Aufgabe ist eine Textaufgabe aus dem Inhaltsbereich Stochastik der siebten Klasse. In der Textaufgabe sollen Alternativen für ein Zufallsgerät gefunden werden, die die gleiche Wahrscheinlichkeit aufweisen. Aus diesem Grund ist eine begriffliche Argumentation notwendig, da unterschiedliche Zufallsgeräte genannt werden müssen, die die gleichen Wahrscheinlichkeiten aufweisen wie das defekte Glücksrad.

Das Glücksrad ist als verwendete mathematische Darstellung (nicht abgebildet) Lerngegenstand, da sich die Wahrscheinlichkeiten durch die Aufteilung am Glücksrad ergeben.

Bei der Aufgabe handelt es sich um eine außermathematische Modellierung. Der Transferbezug kann als konstruiert betrachtet werden, da die Verwendung von anderen Zufallsgeräten in keinerlei Weise praktikabler wäre als das gleiche Glücksrad zu kaufen oder es zu reparieren.

Aufgabe 2 - komprimierend

Mit dem Glücksrad rechts sollten Gewinne bei einem Klassenfest ausgelost werden. Da es defekt ist, soll die Auslosung mit einem anderen Zufallsgerät erfolgen. Nenne mehrere Möglichkeiten (Griesel et al., 2016, S. 194).

In der Aufgabe wird unpersönlich interagiert; der Fokus liegt bei der Betrachtung des Zufallsgerätes und möglicher Alternativen. Der Kern der Aufgabenstellung wurde, wie häufig bei Textaufgaben, als Imperativ formuliert.

Die Aufgabe zeichnet sich durch mehrere Strukturen aus: Es gibt einen Aufgabenstamm, in dem der Kontext und die Aufgabenstellung beschrieben werden, und daneben die Darstellung des Glücksrads.

Für die beiden exemplarisch dargestellten Textaufgaben aus dem komprimierenden Faktor haben Darstellungen als Lerngegenstand eine zentrale Bedeutung. Die Informationen und Daten sind zwar problemlos zu entnehmen, doch wird eine begriffliche Argumentation verlangt. Außerdem sind die Aufgaben in mehrere Strukturen unterteilt - besonders die Text-Bild-Unterteilung ist ein auffälliges 
Charakteristikum. Darüber hinaus sind beide exemplarischen Beispiele unpersönlich dargelegt; es findet außer bei den im Imperativ formulierten Aufgabenaufforderungen keine Interaktion mit den Lesenden durch z. B. den Einbezug von handelnden Personen statt.

\subsubsection{Faktor: beschreibend}

Die erste Textaufgabe des beschreibenden Faktors ist eine Berechnungsaufgabe aus der fünften Klasse. In der Textaufgabe müssen neben der Berechnung der Zuordnung zwischen Arbeitsstundenkosten und Reparaturdauer für unterschiedlich viele Stunden ebenfalls die Anfahrtskosten addiert werden. Bei der zweiten Teilaufgabe ist eine Umkehrrechnung der ersten Teilaufgabe gefragt. Beide Teilaufgaben erfordern nur die rechnerische Lösung der Textaufgabe und damit sind rechnerische Argumente ausreichend.

In der Aufgabe werden Darstellungen (nicht abgebildet) als Illustration verwendet und es muss außermathematisch modelliert werden. Der genutzte Transferbezug ist hierbei als realitätsnah einzustufen. Die Hilfestellung über einen Informationstechnik-Dienstleister und die folgende Abrechnung stehen zwar nicht im direkten Lebensweltbezug der Lernenden, können aber als vorstellbar betrachtet werden.

Aufgabe 1 - beschreibend

Herr Scholz betreut den Computerraum in der Schule. Diesmal hat er ein Problem, das er nicht alleine in den Griff bekommt. Er ruft einen Fachmann an, erkundigt sich aber vorher nach den Preisen. Die Anfahrtskosten betragen 25 Euro, für die Arbeitsstunde berechnet der Computerfachmann 32 Euro.

Wie viel muss Herr Scholz bezahlen, wenn die Reparatur 1, 2, 3 oder 4 Stunden dauert?

Der Computerfachmann muss am nächsten Tag wiederkommen. Wie viele Stunden hat er gearbeitet, wenn die Rechnung dann 242 Euro beträgt? Schreibe dazu eine passende Gleichung (Borneleit \& Winter, 2006, S. 85).

Die Daten und Informationen können aus der Aufgabe problemlos entnommen werden. Dies liegt insbesondere daran, dass keine unnötigen Zahlen, aus denen die Lernenden selektieren müssen, vorhanden sind. 
Bezüglich der Interaktion wird in der Aufgabe eine deutlich beschreibende und persönliche Interaktion verwendet. Dabei wird explizit gemacht, welches Problem vorliegt. Daraus ergeben sich einige Sätze, die für die Aufgabenstellung als redundant betrachtet werden können.

Die erste Textaufgabe aus dem beschreibenden Faktor besitzt mehrere Aufgabenstellungen, die sich in eine Beschreibung des Kontextes der Textaufgabe und die Aufgabenstellung untergliedern.

Das zweite Beispiel aus dem beschreibenden Faktor ist eine Textaufgabe aus dem Bereich der Prozentrechnung aus der siebten Klasse. In der Aufgabe muss der Rabatt für ein Fahrrad berechnet werden. Der errechnete Wert muss angegeben werden, daher sind nur rechnerische Argumente notwendig. In der Textaufgabe werden keine Darstellungen verwendet, weder in Form von Illustrationen noch als Lerngegenstände.

Aufgabe 2 - beschreibend

Alexander will ein City-Bike kaufen. Es kostet 140,00 Euro. Da es sich um ein Modell aus dem Vorjahr handelt, wird der Preis um $15 \%$ herabgesetzt. Wie viel muss Alexander bezahlen? (Griesel et al., 2016, S. 76).

Des Weiteren handelt es sich um eine außermathematische Aufgabe zum Modellieren. Der Transferbezug kann als realitätsnah betrachtet werden, da Rabatte typischerweise im Kontext von Konsumverhalten vorkommen.

Die Entnahme der Daten und Informationen ist unkompliziert. Die beiden Zahlenwerte in der Aufgabe sind zur Berechnung der Aufgabe ausreichend und werden in jeweils zwei Sätze gegliedert. Prinzipiell sind die Entnahme und Berechnung der Textaufgabe ohne das Lesen des Textes möglich.

In der Textaufgabe wird durch den Einbezug einer handelnden Person versucht, eine Interaktion aufzubauen. Dabei dient Alexander als Protagonist in der Aufgabe, die durch eine einzelne Struktur formatiert ist.

Die beiden Beispiele des beschreibenden Faktors deuten darauf hin, dass rechnerische Argumente für diese Gruppe an Aufgaben eher typisch sind. Außerdem kommt eine persönliche Einbettung innerhalb eines realitätsbezogenen Kontextes vor. Die Daten und Informationen sind problemlos aus diesem Kontext zu entnehmen. Weniger Bedeutung scheinen Darstellungen für die Textaufgaben zu besitzen. 


\subsubsection{Faktor: informativ}

Die erste Textaufgabe des informativen Faktors ist eine Prozentaufgabe, für die eine begriffliche Argumentation benötigt wird, die zur Beurteilung der Aussagen im Text dient.

$\mathrm{Da}$ es sich bei der Textaufgabe um eine Meldung aus einer Zeitung handelt, sind keine mathematischen Darstellungen in der Aufgabe vorhanden.

Aufgabe 1 - informativ

Fuhr vor einigen Jahren noch jeder zehnte Autofahrer zu schnell, so ist es mittlerweile heute ,nur noch' jeder fünfte. Doch auch fünf Prozent sind zu viele, und so wird weiterhin kontrolliert, und die Schnellfahrer haben zu zahlen. Nimm Stellung zu den Angaben in der Zeitungsmeldung (Aufgabe aus der PALMA-Studie).

Es handelt sich um eine außermathematische Aufgabe, die einen authentischen Transferbezug zeigt, da es sich beim Kontext der Aufgabe um den angesprochenen Zeitungsartikel handelt, in dem eine falsche Interpretation der Aussage jederfünfte beschrieben werden soll.

Die für die Aufgabe benötigten Informationen sind aus dem Abschnitt zu übernehmen. Es ist aber schwierig zu deuten, welche Informationen zur Stellungnahme wirklich benötigt werden. Der Zusammenhang zwischen der Aussage des ersten Satzes und der fehlerhaften Implikation des zweiten Satzes wird zwar durch die Verwendung der Anführungsstriche verdeutlicht, jedoch ist von einer komplexen Entnahme der Informationen auszugehen.

Die Interaktion im Aufgabentext ist unpersönlich. Es kommen weder Hauptpersonen vor, noch wird direkt die Rezipientin oder der Rezipient angesprochen (bis auf den Imperativ in der Aufgabenstellung). Es handelt sich um eine informierende Meldung ohne persönlichen Bezug.

Die Aufgabe ist durch die Aufteilung in den Zeitungsartikel und die Aufgabenstellung getrennt, wird jedoch nur durch eine einfache Struktur abgebildet.

Die zweite ausgewählte Aufgabe für die Fallanalyse aus dem informativen Faktor ist eine Aufgabe aus der sechsten Jahrgangsstufe und mit dem Inhalt der Dezimalzahlen. In der Aufgabe sind begriffliche Argumente nötig, die das Verständnis der Struktur der Dezimalzahlen hinter dem Komma erfordern. Zwar bietet sich auch eine simple rechnerische Argumentation aufgrund der nicht durch Verben operationalisierten Aufgabenstellung an - zumindest für die erste Teilaufgabe - jedoch ist die Intention der Aufgabenstellung deutlich die einer begrifflichen Argumentation. 
Die Aufgabe orientiert sich an einem Zeitungsinterview. Daher werden für die Textaufgabe keine Darstellungen verwendet. Darüber hinaus wird für die Aufgabe das außermathematische Modellieren mit einem authentischen Transferbezug benötigt, da die Aussagen aus Medien reflektiert werden müssen.

Aufgabe 2 - informativ

Als ein Skirennen einmal mit nur einer hundertstel Sekunde Vorsprung gewonnen wurde, fragte ein Reporter des österreichischen Senders Ö3 Leute auf der Straße: ,Aus wie viel hundertstel Sekunden besteht eine Sekunde?' Hier sind drei der Antworten: ,Tausend, glaube ich, oder? 'Sechzig.' ,Normalerweise ist das so. Eine Minute hat sechzig Sekunden, aber beim Rennen, glaube ich, sind es hundert. Hundertstel Sekunde ist dann das Zehnfache.

a) Wie viele hundertstel Sekunden hat denn jetzt eine Sekunde?

b) Was habend die Leute gedacht? Was hat sie bei ihren Antworten wohl verwirrt?

(B.-J. Frey et al., 2016, S. 17).

Die Interaktion der Aufgabe ist unpersönlich. Zwar wird ein Reporter erwähnt, aber Hauptpersonen spielen in den Darstellungen der Meinungen keine Rolle. Dies wird auch in der zweiten Aufgabenstellung deutlich, indem von Leuten gesprochen wird.

Die Aufgabenstellung zeichnet sich durch eine gegliederte Struktur aus, die die Darstellung des Settings, die Aussagen und die Aufgabenstellung trennt. Die Entnahme der Daten und Informationen kann ebenfalls als schwierig erachtet werden, da sie nicht direkt möglich ist, sondern bereits Voraussetzungen (Kenntnis der Struktur der Dezimalzahlen hinter dem Komma) erfüllt sein müssen, um die Aufgabe zu lösen.

Die beiden genannten Beispiele aus dem informativen Faktor deuten darauf hin, dass für die Gruppe der Textaufgaben eher begriffliche Argumente von Bedeutung sind, die in einen realitätsbezogenen Kontext eingebunden sind. Die Entnahme der Informationen und Daten ist nicht eindeutig und es wird in den Beispielen deutlich, dass Kenntnisse vorausgesetzt werden. Darstellungen haben in den Beispielen keine Relevanz.

\subsubsection{Faktor: instruktiv}

Die erste charakteristische Aufgabe des instruktiven Faktors ist eine Proportionalitätsaufgabe aus der siebten Klasse. In der Aufgabe wird eine Zuordnung 
vorgegeben, die in einem Koordinatensystem eingezeichnet werden soll. In der zweiten Aufgabenstellung soll nach einer Preiserhöhung wiederum eine Zuordnung in das Koordinatensystem gezeichnet werden. Die beiden Graphen sollen verglichen werden. Anhand der Fragestellung kann die Textaufgabe weder der rechnerischen noch der begrifflichen Argumentation zugeordnet werden.

Zentral für die Aufgabenlösung sind die Zeichnung und das Vergleichen. In der Aufgabenstellung werden keine Darstellungen verwendet, es wird aber die Produktion einer Darstellung in Form eines Graphen gefordert.

Aufgabe 1 - instruktiv

$500 \mathrm{~g}$ Schafskäse kosten 5,50 Euro. Zeichne für die Zuordnung Gewicht $\rightarrow$ Preis einen Graphen in ein Koordinatensystem (Rechtsachse: $1 \mathrm{~cm}$ für $100 \mathrm{~g}$; Hochachse: $1 \mathrm{~cm}$ für 1 Euro).

a) Nach einer Preiserhöhung kosten 500 g Schafskäse 6,40 Euro. Zeichne den zugehörigen Graphen in das gleiche Koordinatensystem. Vergleiche die Graphen.

b) Ein Supermarkt bietet den gleichen Schafkäse an. $500 \mathrm{~g}$ kosten dort 4,80€. Zeichne auch hier den zugehörigen Graphen mit den anderen Graphen (Griesel et al., 2016, S. 32).

Im Aspekt der mathematischen Tätigkeit wird eine außermathematische Modellierung verlangt, die sich in Bezug auf den Transferbezug als deutlich konstruiert präsentiert. Die Entnahme der Daten und Informationen gestaltet sich unkompliziert, da die Zahlenwerte offensichtlich vorliegen und zusätzliche Informationen, die die genaue Erklärung zur Zeichnung des Graphen umfassen, dargeboten werden.

Daneben zeichnet sich die Aufgabe durch eine unpersönliche Interaktion aus, wobei die Aufgabenstellung als Imperativ formuliert ist. Die Aufgabe ist durch die zwei Aufgabenstellungen in mehreren Strukturen gegliedert.

Das zweite Aufgabenbeispiel für den instruktiven Faktor ist eine Textaufgabe aus dem Bereich der Rechnung mit natürlichen Zahlen. Für die Aufgabe sind rechnerische Argumente ausreichend. Die Aufgabenstellung ist jedoch unklar formuliert, so wird nur von den Ausgaben der Fußballfans gesprochen. Damit ist nicht eindeutig klar, dass hierbei die Gesamtausgaben gemeint sind.

Für die Aufgaben werden keine weiteren mathematischen Darstellungen verwendet, auch werden keine weiteren Illustrationen genutzt, wie beispielsweise die Abbildung des Stadions.

Aufgabe 2 - instruktiv 
Das Fußballstadion in Dortmund hat 55300 Sitzplätze und ca. 27600 Stehplätze. Wenn das Stadion ausverkauft ist, hat jeder Fußballfan durchschnittlich 40 Euro für das Spiel ausgegeben. Berechne überschlagsmäßig die Ausgaben der Fußballfans (Borneleit \& Winter, 2006, S. 68).

Die Aufgabe ist eine außermathematische Modellierungsaufgabe, die einen konstruierten Transferbezug aufweist. Zwar kann die generelle Rahmung als realitätsnah erachtet werden, da es nicht abwegig ist, Gesamtkosten zu berechnen. Der Kontext der Textaufgabe basiert aber nicht auf einem dementsprechend realitätsnahen Aspekt, sondern fordert nur die überschlagsmäßige Berechnung, deren Sinn in der Textaufgabe unklar bleibt.

Die Interaktion in der Textaufgabe ist unpersönlich, mit imperativer Verwendung der Aufgabenstellung. Dabei präsentiert sich die Aufgabe in einer einzelnen Struktur des Aufgabenstamms.

Die beiden exemplarischen Textaufgaben aus der Gruppe des instruktiven Faktors weisen darauf hin, dass der Realitätsbezug bei den Textaufgaben vorhanden, jedoch eher konstruiert ist und keine Interaktion zwischen Personen aufweist. Die Daten und Informationen sind aufgrund der meist klaren Darstellung der notwendigen Werte problemlos zu entnehmen. Die Aufgabenstellungen sind in einer unkomplizierten Struktur dargestellt, ohne starke Abhebungen von Aufgabenstellungen oder weiteren Elementen der Textaufgabe. Weniger bedeutend ist die Verwendung von Darstellungen.

\subsection{Bildung von Aufgabentypen}

Die fallspezifische Analyse in Abschnitt 9.2 hat demonstriert, dass sich für die charakteristischen Aufgaben der Faktoren inhaltliche und kontextuelle Besonderheiten ergeben. Neben der Fallinterpretation ist das weitere Ziel der qualitativen Vertiefungsanalyse die Bildung von Aufgabentypen, die die in Abschnitt 9.2 gezeigten inhaltlichen und kontextuellen Besonderheiten für alle Fallbeispiele je Faktor verallgemeinern und mit einer Bezeichnung hinterlegen. Die Bezeichnung soll darstellen, welche fachlichen und kontextuellen Besonderheiten sich für die Aufgaben eines Faktors ergeben und wie sich die Aufgaben von denen der anderen Faktoren unterscheiden. Als Indikator für die fachlichen und kontextuellen Besonderheiten wird die häufigste Kodierung der Subkategorien in einer Hauptkategorie verwendet. Da sich die Aufgaben der Faktoren in der Hauptkategorie Daten und Informationen nicht in der häufigsten Kodierung (wohl aber in den Einzelfällen) unterscheiden, wird zur Darstellung und Interpretation 
auf diese Hauptkategorie zur Typenbildung verzichtet. Basis der Interpretation ist das durch das deduktiv-induktive Vorgehen erstellte Kategoriensystem (vgl. Abschnitt 9.1.3). Es werden zur Interpretation sowohl theoretische Aspekte, die für die deduktiv erstellten Kategorien verwendet wurden, als auch explorative Aspekte, die für die induktiven Kategorien gebildet wurden, genutzt.

Überblick (Abschnitt 9.3): Wie die Textaufgaben der Fallanalyse wurden auch die restlichen ausgewählten Textaufgaben analysiert und kodiert. Ergänzend zur Fallanalyse soll über die häufigste Kodierung angezeigt werden, welche fachlichen und kontextuellen Kategorien insbesondere für die Textaufgaben eines Faktors vorkommen. Damit kann die fallspezifische Typisierung auf alle $N=49$ Textaufgaben verallgemeinert werden, die für die Faktoren betrachtet werden. Dies wird für den erklärenden (Abschnitt 9.3.1), komprimierenden (Abschnitt 9.3.2), beschreibenden (Abschnitt 9.3.3), informativen (Abschnitt 9.3.4) und instruktiven (Abschnitt 9.3.5) Faktor dargestellt. Um Aufgabentypen zu beschreiben, benötigt es neben der Darstellung der individuellen Besonderheiten einen kontrastierenden Vergleich der häufigsten Kodierung der Subkategorien. Die Kontrastierung dient der Unterscheidung zwischen den Textaufgaben der jeweiligen Faktoren. Dadurch wird deutlich, was das Typische der Textaufgaben ist, aus dem sich die Typisierung und die Bezeichnung der Textaufgaben ableiten lässt (Abschnitt 9.3.6).

\subsubsection{Faktor: erklärend}

In Anbetracht der häufigsten Kodierung der Subkategorien je Hauptkategorie für die $N=10$ Textaufgaben des erklärenden Faktors ergeben sich bestimmte Ausprägungen. Das Ergebnis der häufigsten Kodierung der Subkategorien ist in Abbildung 9.3 dargestellt. In der und den darauffolgenden Abbildungen sind die (nominalskalierten) Kodierungen in einem Koordinatensystem abgebildet. Dargestellt ist die häufigste Kodierung der Subkategorien je Hauptkategorie der Textaufgaben.

Wie im Abschnitt 9.2.1 bereits angedeutet, sind gegliederte Strukturen ein auffälliges Spezifikum der Aufgaben des erklärenden Faktors. Die häufigste Kodierung in der Hauptkategorie für die Aufgaben war die gegliederte Struktur (3), in der Abbildung 9.3 in blaugrün dargestellt (Gestalt). Die Subkategorie begriffliche Argumente (3) wird am häufigsten in der Hauptkategorie mathematische Argumentation (Argumentation - lila) kodiert. 
Neben der gegliederten Struktur erhalten Aufgaben aus dem erklärenden Faktor am häufigsten die Kodierung unpersönlich (2) für die Hauptkategorie Interaktion (in dunkelgrün).

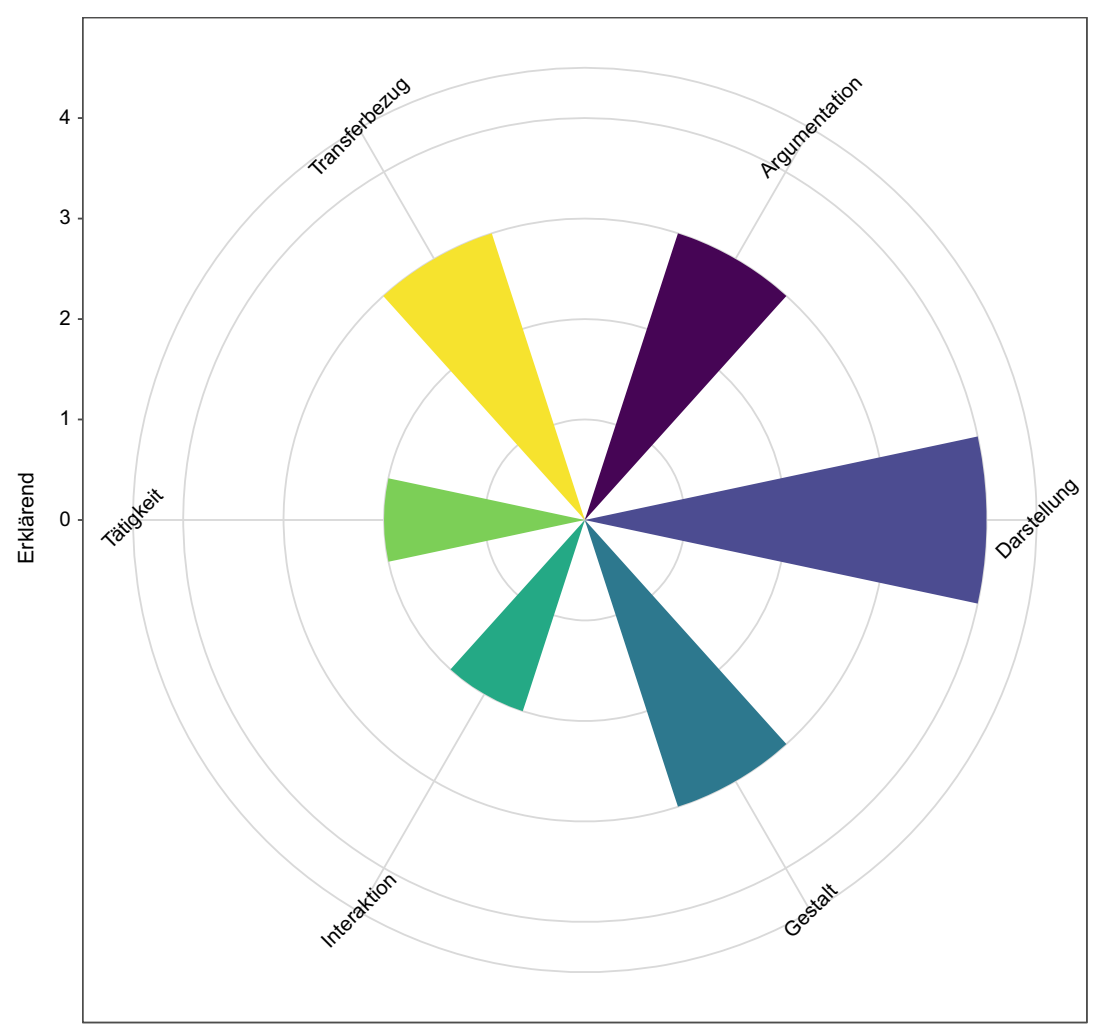

Hauptkategorie

Abbildung 9.3 Darstellung der häufigsten Kodierung der Hauptkategorien bei Aufgaben aus dem Faktor: erklärend (Eigene Erstellung)

Die häufigste Kodierung in der Hauptkategorie Transferbezug (gelb) erhalten die Textaufgaben des erklärenden Faktors in der Subkategorie realitätsnah oder authentisch (3). Die häufige Kodierung in der Hauptkategorie Tätigkeit (hellgrün) erhält die Subkategorie des außermathematischen Modellierens (2). Am 
häufigsten in der Hauptkategorie mathematische Darstellungen (Darstellungen), in dunkelblau, wurde die Subkategorie Darstellungen als Lerngegenstand (4) kodiert.

Die Aufgaben des erklärenden Faktors, in denen außermathematisches Modellieren in realitätsnahen und authentischen Sachverhalten verlangt wird, zeichnen sich durch die besondere strukturelle Gestalt aus. Durch die gegliederte Struktur werden in den Aufgaben situativer Kontext, Daten und Informationen sowie Darstellungen und Aufgabenstellung deutlich voneinander getrennt. Für diese Aufgaben deutet sich damit an, dass die Ausprägung in Bezug auf die gegliederte Struktur als typisch zu betrachten ist. Darüber hinaus sind der Bezug zum außermathematischen Modellieren mit realitätsnahen und authentischen Aufgaben sowie die Verbindung mit Darstellungen als Lerngegenstand häufig. Außerdem sind begriffliche Argumente für die Aufgabenlösung notwendig und lassen sich als Charakteristika für die Gruppe der Textaufgaben des erklärenden Faktors benennen.

\subsubsection{Faktor: komprimierend}

Hinsichtlich der häufigsten Kodierung der Hauptkategorien zeigen sich Spezifika der $N=10$ Textaufgaben, die in der qualitative Vertiefungsanalyse für den komprimierenden Faktor betrachtet wurden. Die häufigste Kodierung der Hauptkategorien ist in Abbildung 9.4 dargestellt. In der Fallanalyse in Abschnitt 9.2.2 wurde deutlich, dass für die beiden Textaufgaben Darstellungen als Lerngegenstand eine zentrale Bedeutung haben. Dies bestätigt sich auch in dieser Vertiefungsanalyse mit den weiteren Textaufgaben des Faktors, bei der ebenfalls diese Subkategorie am häufigsten kodiert wurde. Die Informationen und Daten sind zwar problemlos zu entnehmen, doch wird eine begriffliche Argumentation verlangt. Außerdem sind die Aufgaben in mehrere Strukturen unterteilt, besonders die Text-Bild-Unterteilung ist ein auffälliges Charakteristikum. Darüber hinaus sind beide Beispiele unpersönlich formuliert, es findet - außer bei den im Imperativ formulierten Aufgabenanforderungen - keine Interaktion mit den Lesenden durch z. B. den Einbezug von handelnden Personen statt.

Wie bereits durch die fallspezifische Analyse angedeutet, haben Darstellungen als Lerngegenstand (4) in der Hauptkategorie Gebrauch von mathematischen Darstellungen (Darstellung - dunkelblau) eine hohe Bedeutung. Ebenfalls verdichten sich die Bedeutungen der innermathematischen Modellierung und der Hauptkategorie mathematische Tätigkeit (Tätigkeit, hellgrün), die für die Aufgaben des komprimierenden Faktors am häufigsten kodiert wurde. Aufgrund der häufigen 
Kodierung der innermathematischen Modellierung ist die häufigste Kodierung in der Kategorie Transferbezug (gelb), dass kein Transferbezug vorhanden ist (1).

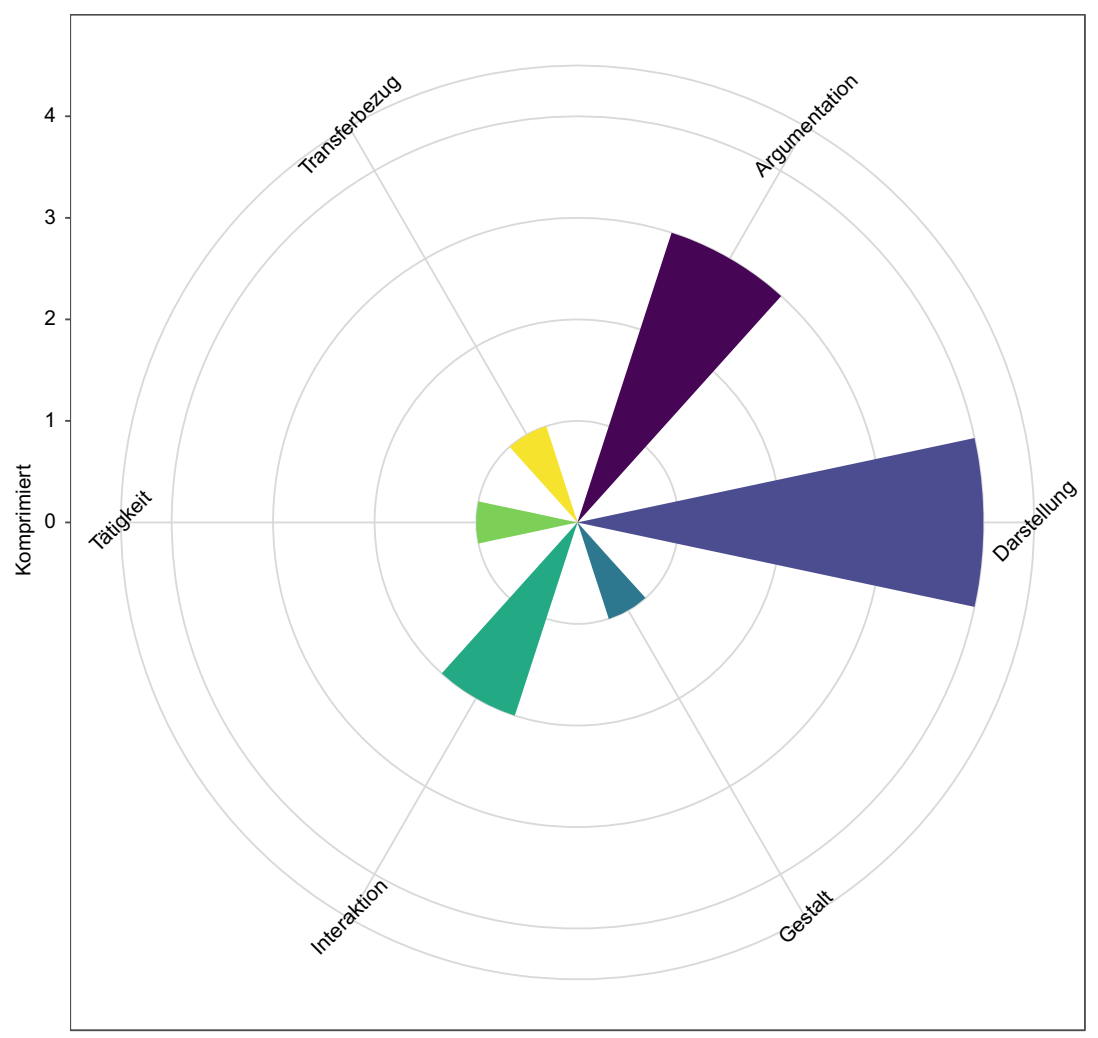

Hauptkategorie

Abbildung 9.4 Darstellung der häufigsten Kodierung der Hauptkategorien bei Aufgaben aus dem Faktor: komprimierend (Eigene Erstellung)

In der Hauptkategorie strukturelle Gestalt (Gestalt, blaugrün) wurde die Subkategorie einzelne Strukturen (1) besonders häufig kodiert. In der Hauptkategorie Interaktion (dunkelgrün) wurde unpersönlich (2) am häufigsten in der Gruppe der Textaufgaben aus dem komprimierenden Faktor kodiert. 
Wie die Fallbeispiele in Abschnitt 9.2.2 bereits dargestellt haben, kann die begriffliche Argumentation (3) in der Hauptkategorie mathematische Argumentation (Argumentation, lila) als typisch für die Textaufgaben betrachtet werden, die dem komprimierenden Faktor zugeordnet sind.

Zusammenfassend lässt sich zu den Textaufgaben des komprimierenden Faktors festhalten, dass sich diese durch mathematische Darstellungen als Lerngegenstand auszeichnen und häufig eine begriffliche Argumentation verlangen. Außerdem charakteristisch für die Textaufgaben des komprimierenden Faktors ist die innermathematische Modellierung ohne Transferbezug, was die häufige unpersönliche Interaktion in den Aufgaben erklärt. Trotz der Relevanz an Darstellungen treten in den Textaufgaben häufig einzelne Strukturen auf.

\subsubsection{Faktor: beschreibend}

Um die Analyse der exemplarischen Textaufgaben für den beschreibenden Faktor in Abschnitt 9.2.3 zu ergänzen und die Ausprägung aller $N=10$ Textaufgaben $\mathrm{zu}$ verdeutlichen, sind in Abbildung 9.5 die häufigsten Kodierungen der Subkategorien von den Hauptkategorien aufgetragen.

Für die Gruppe der Textaufgaben des beschreibenden Faktors ist die rechnerische Argumentation (2) häufig in der Hauptkategorie mathematische Argumentation (Argumentation, lila). Aufgrund der hohen Relevanz von rechnerischen Argumentationen scheint die Bedeutung für Darstellungen in den Textaufgaben für diesen Faktor gering. So ist die Kodierung keine Darstellungen (1) in der Hauptkategorie mathematische Darstellungen (Darstellung, dunkelblau) am häufigsten. In der Hauptkategorie strukturelle Gestalt (Gestalt, blaugrün) wurde die Subkategorie einzelne Strukturen häufig kodiert.

Der Transferbezug (3) ist häufig realitätsbezogen und authentisch, dahingehend handelt es sich in der Hauptkategorie mathematische Tätigkeit (Tätigkeit, hellgrün) häufig um eine außermathematische Modellierung. In diesem Zusammenhang werden die Textaufgaben des beschreibenden Faktors in der Hauptkategorie Interaktion (dunkelgrün) am häufigsten mit persönlich (1) kodiert.

Hinsichtlich der häufigsten Kodierung lassen sich die Aufgaben des beschreibenden Faktors als Textaufgaben charakterisieren, für die die rechnerische Argumentation eine hohe Bedeutung besitzt, die dazu führt, dass die Aufgaben meist eine wenig ausdifferenzierte Gestalt aufweisen und dass Darstellungen keine Bedeutung für diese Aufgaben haben. Außerdem zeigen die Textaufgaben häufig 


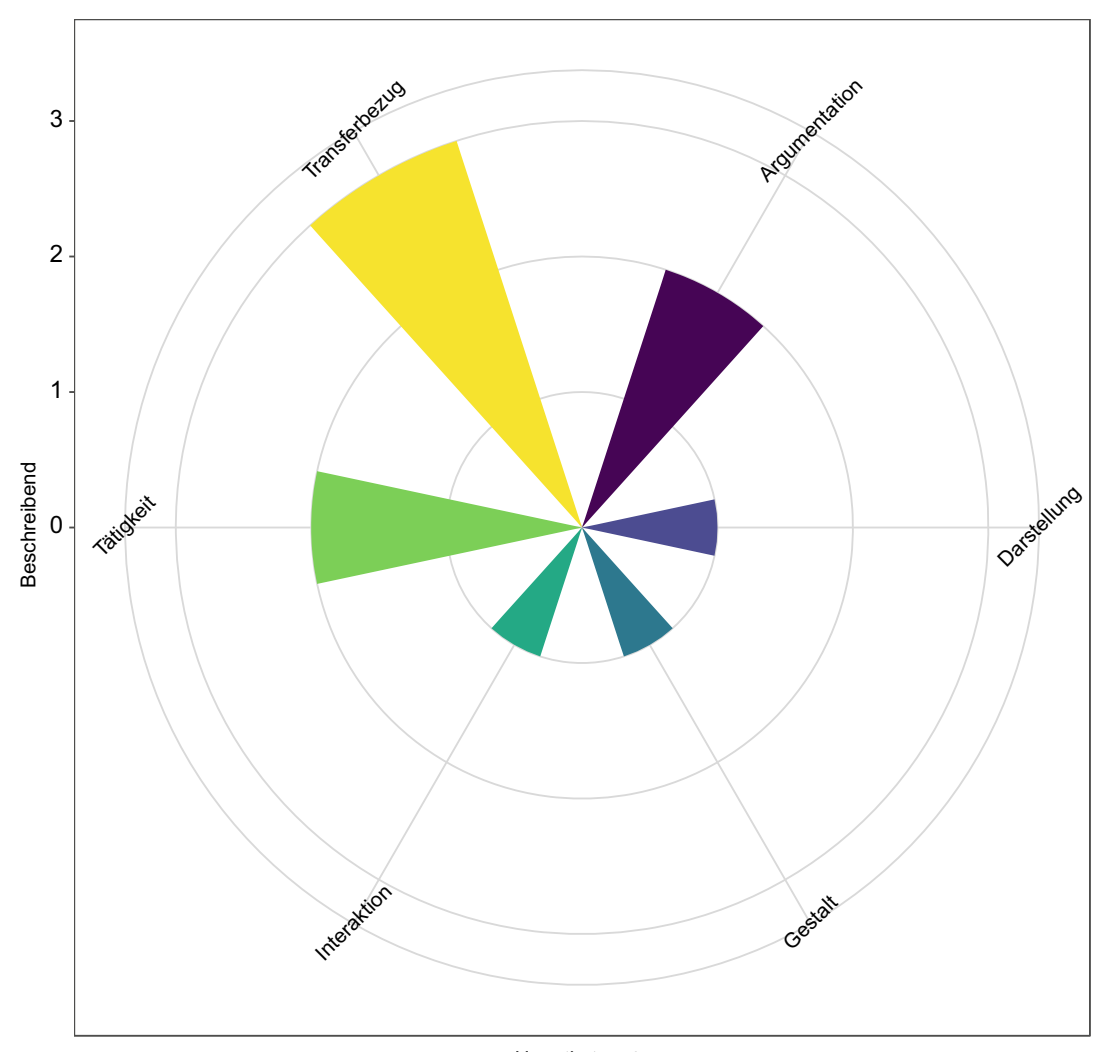

Hauptkategorie

Abbildung 9.5 Darstellung der häufigsten Kodierung der Hauptkategorien bei Aufgaben aus dem Faktor: beschreibend (Eigene Erstellung)

einen Realitätsbezug und die Notwendigkeit der außermathematischen Modellierung des Gegenstandes. Für den Realitätsbezug wird häufig eine persönliche Gestaltung genutzt, in der beispielsweise Personen dargestellt werden.

\subsubsection{Faktor:informativ}

Zur Darstellung der Spezifika der $N=9$ Textaufgaben des informativen Faktors sind in Abbildung 9.6 die häufigsten Kodierungen der Hauptkategorien abgebildet. 
Die Textaufgaben des informativen Faktors erhalten in der Hauptkategorie mathematische Argumentation (Argumentation, lila) häufig die Kodierung begriffliche Argumentation (3).

Wie bereits in den Fallbeispielen in Abschnitt 9.2.4 verdeutlicht, haben Darstellungen häufig keine Bedeutung für die Textaufgaben des informativen Faktors. Die häufigste Kodierung in der Hauptkategorie Gebrauch von mathematischen Darstellungen (Darstellung, dunkelblau) erhält die Subkategorie keine Darstellungen (1). Daneben werden die Textaufgaben in der Hauptkategorie strukturelle Gestalt häufig mit einzelnen Strukturen (1) kodiert.

Die Hauptkategorie Transferbezug (gelb) erhält meist die Kodierung realitätsbezogen oder authentisch (3). Die Textaufgaben erhalten in der Hauptkategorie mathematische Tätigkeit häufig eine Kodierung des außermathematischen Modellierens (2). Die Hauptkategorie Interaktion wird häufig mit unpersönlich (2) kodiert.

Die $N=9$ ausgewählten charakteristischen Textaufgaben für den informativen Faktor zeichnen sich durch eine häufige Verwendung von begrifflicher Argumentation aus. Diese begriffliche Argumentation ist unabhängig von verwendeten Darstellungen, die meist keine Relevanz haben. Der Aufbau der Textaufgaben ist zumeist unkompliziert ohne spezifische Gliederung der Elemente der Textaufgabe. Außerdem sind für die Aufgaben der Transferbezug häufig mit einer außermathematischen Modellierung verbunden. Die Darstellung des Gegenstands wird ohne Bezug auf Hauptpersonen geleistet und ist meist unpersönlich.

\subsubsection{Faktor: instruktiv}

Unter der Perspektive der Darstellung typischer Ausprägungen der Kodierung für die $N=10$ Textaufgaben, die für den instruktiven Faktor für die qualitative Vertiefungsanalyse betrachtet wurden, sind in Abbildung 9.7 die häufigsten Kodierungen dargestellt.

In der Hauptkategorie mathematische Argumentation (Argumentation, lila) erhält die rechnerische Argumentation (2) die häufigste Kodierung. Häufig werden in den Textaufgaben aus dem instruktiven Faktor keine Darstellungen (1) in der Hauptkategorie Gebrauch von mathematischen Darstellungen (Darstellung, dunkelblau) genutzt. Die Hauptkategorie strukturelle Gestalt (Gestalt, grünblau) wird am häufigsten mit der Subkategorie einzelne Strukturen (1) kodiert.

Meist kann den Textaufgaben in der Hauptkategorie Transferbezug (gelb) kein Transferbezug zugeordnet (1) werden. Häufig handelt es sich bei den Textaufgaben um außermathematische Modellierungsaufgaben (2) der Hauptkategorie 


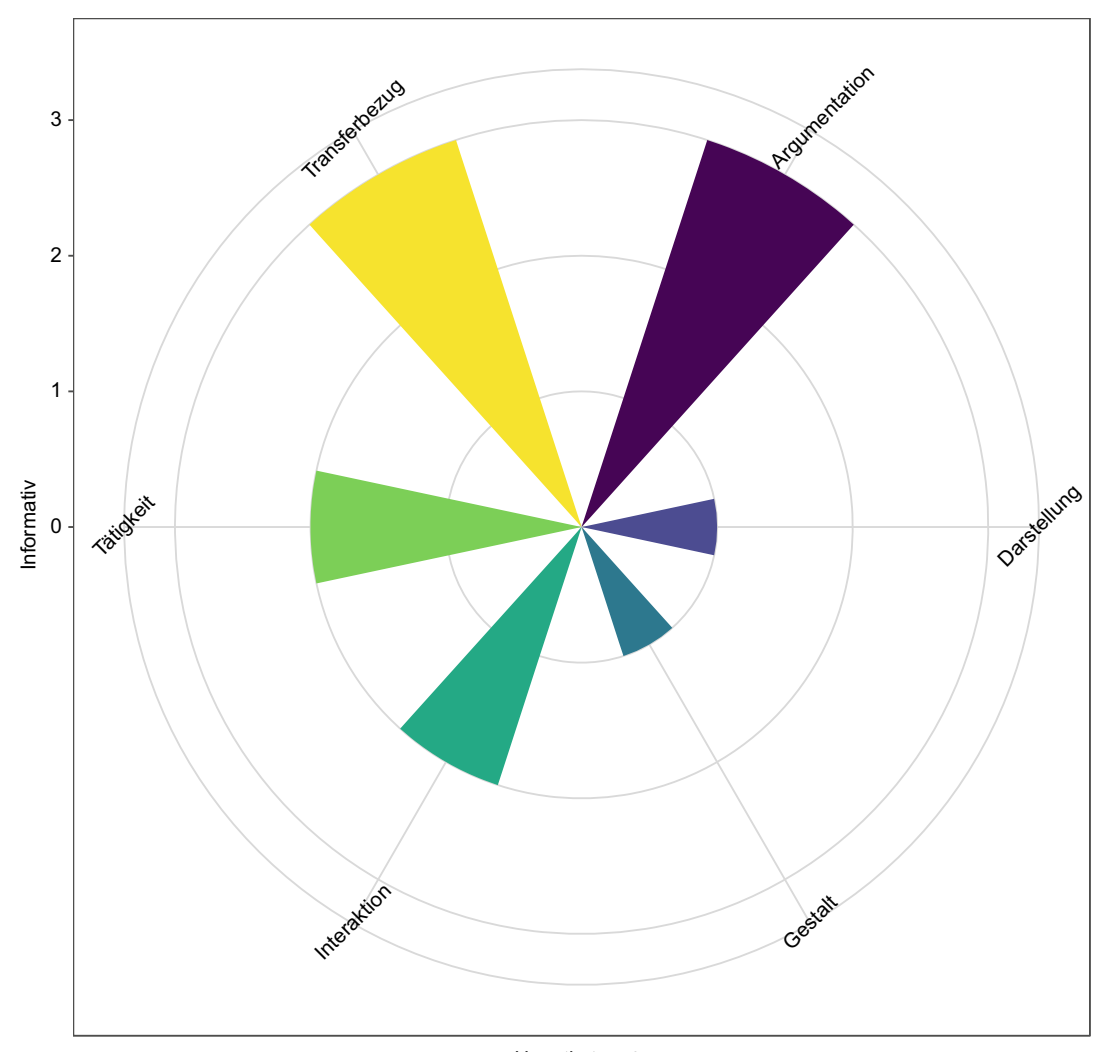

Hauptkategorie

Abbildung 9.6 Darstellung der häufigsten Kodierung der Hauptkategorien bei Aufgaben aus dem Faktor: informativ (Eigene Erstellung)

mathematische Tätigkeit (Tätigkeit, hellgrün). Die Hauptkategorie Interaktion wird häufig mit unpersönlich (2) kodiert.

Für die Textaufgaben des instruktiven Faktors stellt sich dar, dass in diesen häufig rechnerische Argumentationen genutzt werden und dafür keine Unterstützung einer mathematischen Darstellung benötigt wird sowie keine besonderen strukturellen Eigenschaften von Trennung zwischen einzelnen Teilen der Aufgabe erforderlich sind. Meist können die Textaufgaben dem außermathematischen Modellieren zugeordnet werden, jedoch kann häufig kein direkter Transferbezug festgestellt werden. Daher ist unklar, ob die Aufgaben eher konstruiert oder 


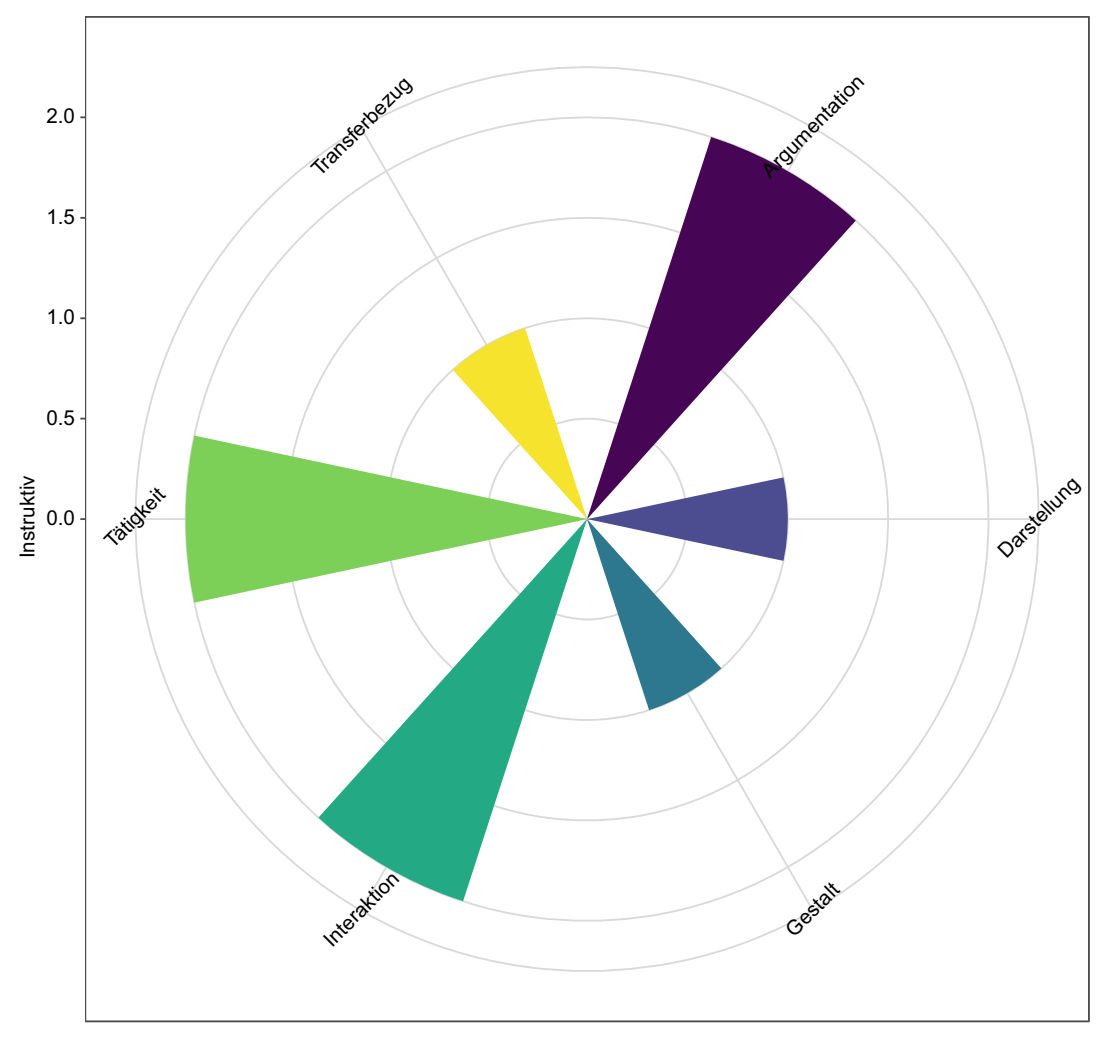

Hauptkategor

Abbildung 9.7 Darstellung der häufigsten Kodierung der Hauptkategorien bei Aufgaben aus dem Faktor: instruktiv (Eigene Erstellung)

realitätsnah und authentisch sind. Darüber hinaus ist die außermathematische Modellierung häufig unpersönlich.

Ausblick: Die häufigste Kodierung der Unterkategorien der Hauptkategorien je Faktor stellt, neben der exemplarischen Analyse, dar, welche typischen Ausprägungen der Kodierung auffällig sind. Nachdem für die einzelnen Textaufgaben der Faktoren gezeigt wurde, was die Aufgaben charakterisiert, soll im anschließenden Abschnitt 9.3.6 durch eine komparative Analyse der häufigsten Kodierungen der 
Unterschied zwischen den Textaufgaben genutzt werden, um eine Bezeichnung zu finden, die die jeweiligen Textaufgaben von den anderen unterscheidet.

\subsubsection{Bildung von Aufgabentypen}

Für die komparative Analyse mit dem Ziel der Bildung von Aufgabentypen wird zum einen die häufigste Kodierung je Faktor ${ }^{1}$ (Textaufgaben des Faktors), dargestellt in Abbildung 9.8, zum anderen die häufigste Kodierung je Hauptkategorie, verdeutlicht in Abbildung 9.9, verwendet und die Kodierungen werden miteinander verglichen.

In Abbildung 9.8 und Abbildung 9.9 sind die Kodierungen jeweils in einem Balkendiagramm abgetragen. Die Abbildung gliedert sich nach Anzahl der Faktoren (5, Abbildung 9.8) oder Hauptkategorien (6, Abbildung 9.9), wobei im oberen Bereich der Überschrift jeweils angegeben ist, um welchen Faktor bzw. welche Hauptkategorie es sich handelt. Skaliert sind die Darstellungen zum einen in der Ordinate nach der jeweiligen Kodierung und in der Abszisse nach der Hauptkategorie bzw. dem Faktor. Wie bereits in Abschnitt 9.3.5 erwähnt, wurde die Hauptkategorie Daten und Informationen ausgeschlossen, da die häufigste Kodierung bei allen Hauptkategorien gleich war.

Die Abbildung 9.8 verdeutlicht im Vergleich mit allen fünf Faktoren, dass es Unterschiede in der Häufigkeit der Kodierung der Subkategorien der Hauptkategorien je Faktor gibt. Zwar haben einige Faktoren gleich häufige Kodierungen, beispielsweise der erklärende, informative und komprimierende Faktor in der Hauptkategorie Argumentieren mit der häufigsten Kodierung der begrifflichen Argumentation (3), jedoch zeigen sich Unterschiede in den Häufigkeiten der Kodierungen für die anderen Subkategorien der Hauptkategorien, etwa in der Interaktion zwischen erklärendem und komprimierendem Faktor, der Darstellung zwischen erklärendem und informativem Faktor sowie dem Transferbezug zwischen informativem und komprimierendem Faktor. Das bedeutet, dass sich alle Faktoren in der Zusammenstellung der Kodierung der Hauptkategorien jeweils untereinander unterscheiden und sich somit besondere Spezifika ergeben, die zur Interpretation einer Bezeichnung für die jeweiligen Textaufgaben je Faktor genutzt werden können.

\footnotetext{
${ }^{1}$ Zur Verkürzung und verbesserten Lesbarkeit, wird die Gruppe der Textaufgaben je Faktor, in diesem Kapitel nur noch Faktor genannt. Gemeint sind jedoch die Kodierungen der Textaufgaben des jeweiligen Faktors.
} 


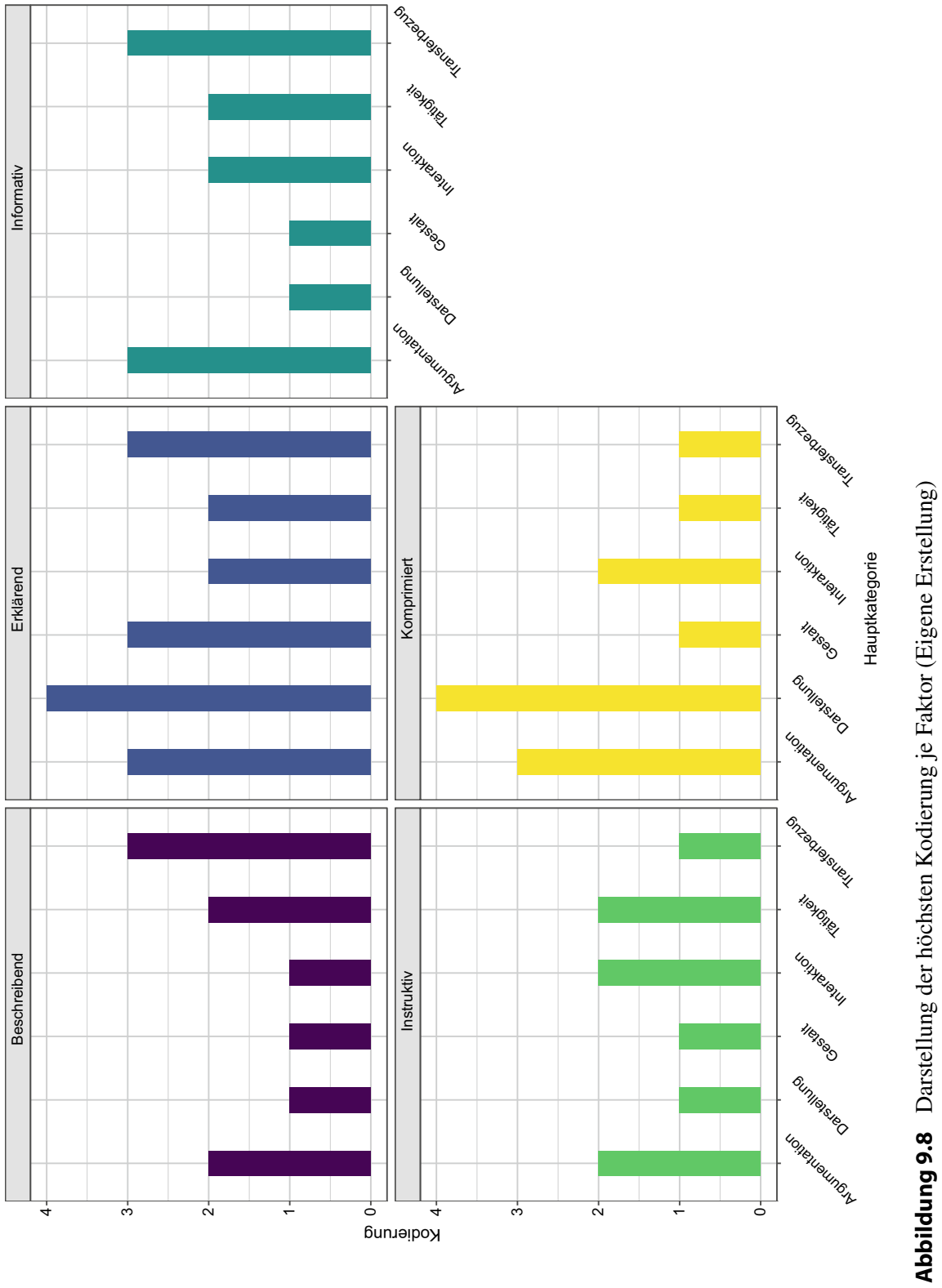


Für eine einzelne Betrachtung der Unterschiede in den Hauptkategorien wird die Abbildung 9.9 genutzt. In dieser ist die Kodierung der Subkategorien der Hauptkategorie je Faktor abgetragen.

In Abbildung 9.9 ist für die einzelnen Hauptkategorien zu erkennen, dass drei Faktoren - als einzige Faktoren - jeweils bei einer Hauptkategorie die häufigste Kodierung einer Subkategorie erhalten. Die erste Hauptkategorie, die sich durch eine einzigartige Häufigkeit der Kodierung auszeichnet, ist die der strukturellen Gestalt (Gestalt, blaugrün). Für die strukturelle Gestalt ist zu erkennen, dass sich ein Balken von den anderen in der häufigsten Kodierung dieser Hauptkategorie unterscheidet. Der Balken entspricht der Zuordnung zum erklärenden Faktor, der häufig mit einer gegliederten Struktur (3) kodiert wurde. Die zweite Hauptkategorie mit einer einzigartig häufigen Kodierung ist die Interaktion (dunkelgrün). In Abbildung 9.9 ist zu erkennen, dass der Faktor beschreibend häufig und als einziger Faktor mit persönlich (1) kodiert wurde. Die dritte Hauptkategorie, in der eine einzelne häufige Kodierung auftritt, ist die mathematische Tätigkeit (Tätigkeit, grün). Für die Hauptkategorie Tätigkeit ist der Faktor komprimierend spezifisch, mit einer häufigen Kodierung der innermathematischen Tätigkeit (1).

Die Textaufgaben des erklärenden Faktors unterscheiden sich spezifisch von den anderen Textaufgaben der Faktoren - besonders durch die strukturelle Gestalt. Die Textaufgaben des beschreibenden Faktors differenzieren sich insbesondere durch die Interaktion. Die Textaufgaben des komprimierenden Faktors zeichnen sich im Vergleich zu den anderen Textaufgaben der Faktoren besonders durch die mathematische Tätigkeit aus. Aufgrund der geschilderten Kodierung, der fallspezifischen Analyse in Abschnitt 9.2. und der Gesamtbetrachtung der häufigsten Kodierung in Abschnitt 9.3 können für die Textaufgaben folgende Bezeichnungen abgeleitet werden:

1. Textaufgaben des erklärenden Faktors: Diese Textaufgaben zeichnen sich besonders durch den strukturellen Aufbau aus. Die meist außermathematische Modellierung mit einem realitätsnahen oder authentischen Sachkontext wird häufig mit Darstellungen verknüpft. Um diese vielfältigen Inhalte sichtbar zu strukturieren, sind die Textaufgaben des erklärenden Faktors in unterschiedliche Sequenzen untergliedert, beispielsweise durch die Trennung von Transferbezug, Darstellungen und Fragestellungen. Aufgrund dessen werden die Textaufgaben zur zusammenfassenden Verdeutlichung mit dem Aufgabentyp sequenzielle Aufgaben bezeichnet (vgl. Tabelle 9.3).

2. Textaufgaben des komprimierenden Faktors: Die Textaufgaben des komprimierenden Faktors lassen sich spezifisch von den anderen Textaufgaben durch 


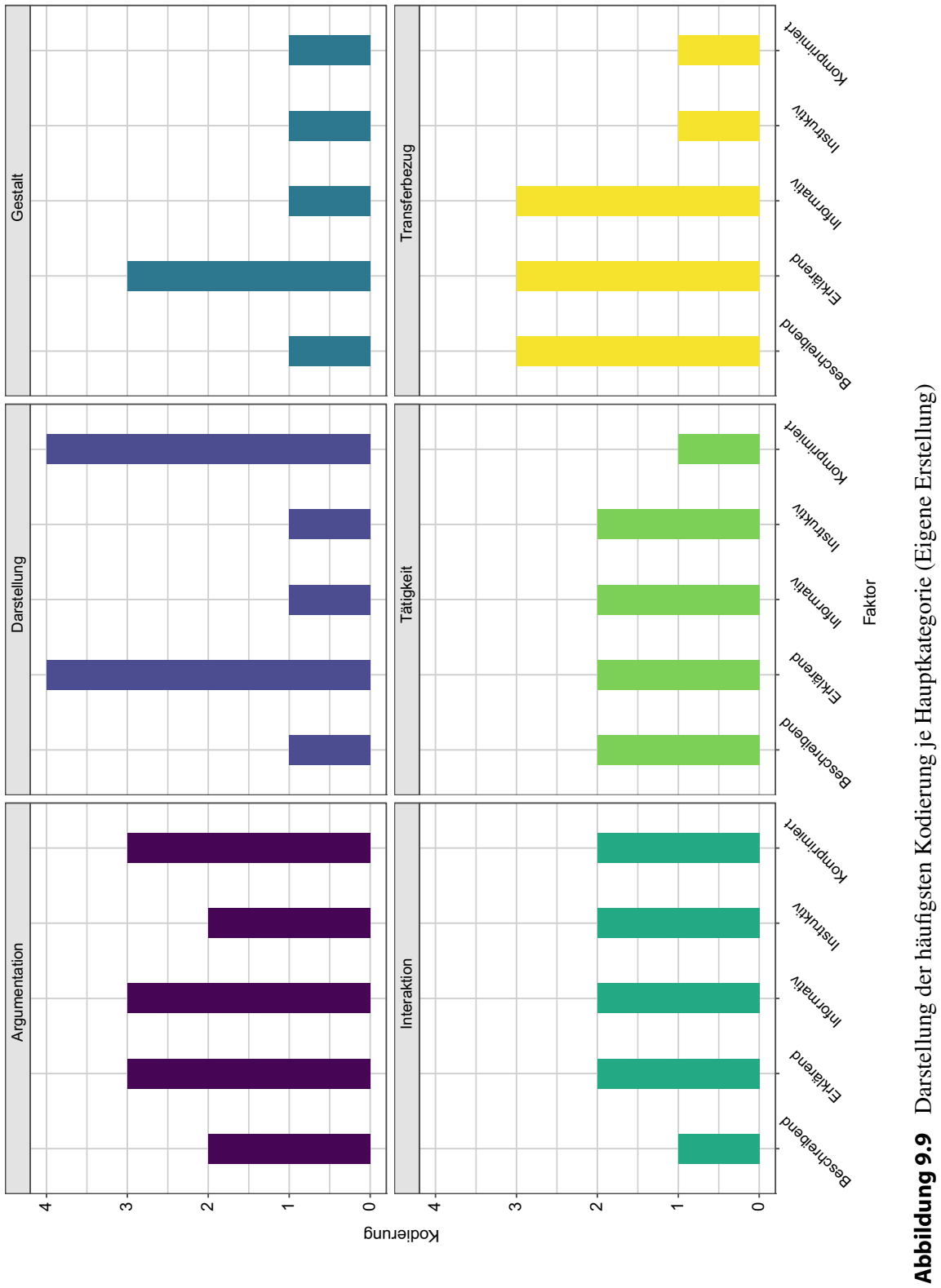


die häufige innermathematische Modellierung unterscheiden. Die innermathematische Modellierung wird häufig im Zusammenhang mit Darstellungen als Lerngegenstand verwendet, was auf die Bedeutung der Darstellungen für die innermathematische Modellierung hinweist, die Gegenstand oder zentraler Aspekt der Aufgabe sind. Typisiert werden die Textaufgaben des komprimierenden Faktors also von den Darstellungen, die durch diese Textaufgaben vermittelt werden sollen. Angesichts dessen werden die Textaufgaben mit dem Aufgabentyp ikonische Aufgaben bezeichnet (vgl. Tabelle 9.3).

3. Textaufgaben des beschreibenden Faktors: Für Textaufgaben des beschreibenden Faktors kommen häufig und spezifisch persönliche Interaktionen vor. Die persönliche Interaktion ist im Rahmen der außermathematischen Modellierung und des realitätsnahen und authentischen Transferbezugs zu interpretieren, der durch die persönliche Interaktion weniger abstrakt dargestellt wird. Die Tendenz dieser Textaufgaben, weniger abstrakt zu sein, ist ebenfalls in der häufigen Kodierung der rechnerischen Argumentation zu erkennen. Die Bedeutung der rechnerischen Argumentation deutet sich als ein wesentliches Charakteristikum an, da häufig der Transferbezug zwar vorhanden, realitätsnah und authentisch, jedoch nicht zwingend notwendig ist, um die Aufgabenstellung zu berechnen. Anhand der Interpretation werden die Textaufgaben des beschreibenden Faktors mit dem Aufgabentyp rechnerische Aufgaben bezeichnet (vgl. Tabelle 9.3).

Für die restlichen Textaufgaben, die keine spezifisch häufige Kodierung hinsichtlich einer Hauptkategorie aufweisen, muss die Bezeichnung des Aufgabentyps aus den anderen häufigen Kodierungen der Hauptkategorien und dem Vergleich mit den anderen Textaufgaben der restlichen Faktoren abgeleitet werden.

Die Herausforderung der Interpretation einer Bezeichnung für die Textaufgaben des informativen Faktors besteht darin, dass diese keine spezifische oder (teil-)spezifische häufige Kodierung (also häufige Kodierung mit einem weiteren Faktor) aufweisen. Entsprechend wird die häufigste Kodierung der Textaufgaben betrachtet und verglichen mit den häufigsten Kodierungen der anderen Textaufgaben der Faktoren. Aus dem Vergleich wird eine Bezeichnung abgeleitet. Es werden besonders die Hauptkategorien mit zwei weiteren, gleich häufigen Kodierungen der Textaufgaben betrachtet. Entsprechend der Abbildung 9.9 werden zur Interpretation insbesondere die Hauptkategorien Argumentation, Darstellung und Transferbezug betrachtet.

Für die Textaufgaben des informativen Faktors ist häufig eine begriffliche Argumentation (3) notwendig. Ebenfalls die häufigste Kodierung in dieser Subkategorie haben die Textaufgaben des erklärenden und komprimierenden Faktors. 
Eine häufige Kodierung weisen auch die Textaufgaben des informativen Faktors für die Hauptkategorie Darstellung und mit der Kodierung keine Darstellungen (1), mit den Faktoren beschreibend und instruktiv auf. Eine weitere häufige Kodierung haben die Textaufgaben des informativen Faktors mit den Textaufgaben des beschreibenden und erklärenden Faktors in der Hauptkategorie Transferbezug (gelb) und der häufigen Kodierung realitätsnah und authentisch (3).

4. Textaufgaben des informativen Faktors: Die Textaufgaben des informativen Faktors zeichnen sich durch häufige begriffliche Argumentationen aus. Diese Argumentation ist nicht wie im Fall der Textaufgaben des komprimierenden Faktors mit einer hohen Bedeutung an die Darstellung geknüpft. Daneben ist keine starke Strukturierung des Inhalts vorhanden, was die Textaufgaben des informativen Faktors von denen des erklärenden Faktors differenziert. Der Transferbezug der Textaufgaben des informativen Faktors unterscheidet sich von dem der Aufgaben des beschreibenden Faktors durch die unpersönliche Interaktion. Verglichen mit den Textaufgaben des instruktiven Faktors unterscheiden sich die Textaufgaben in der begrifflichen statt rechnerischen Argumentationen. Der realitätsnahe und authentische Transferbezug mit der begrifflichen Argumentation deutet darauf hin, dass die Inhalte der Textaufgabe in der Aufgabenlösung eine hohe Relevanz besitzen und nicht nur zur direkten und offensichtlichen Entnahme von Zahlen und Daten dienen. Die geringe Bedeutung von Darstellungen weist ebenfalls auf die Relevanz des in den Aufgaben formulierten Gegenstands (Sache) hin. Die Interpretation der Spezifika der Textaufgaben des informativen Faktors führt zu der Bezeichnung des Aufgabentyps sachliche Aufgaben (vgl. Tabelle 9.3).

Für die Bezeichnung der Textaufgaben des instruktiven Faktors wird insbesondere der komparative Vergleich zwischen diesen und dem beschreibenden und komprimierenden Faktor dargestellt. So teilen der verbleibende instruktive Faktor (teil-)spezifisch häufige Kodierungen bei gewissen Hauptkategorien mit dem beschreibenden Faktor. In Abbildung 9.9 ist dargestellt, dass sich der instruktive Faktor eine (teil-)spezifisch häufige Kodierung mit dem beschreibenden Faktor in der Hauptkategorie mathematische Argumentation (lila) teilt. Die Textaufgaben beider Faktoren wurden häufig mit rechnerischer Argumentation (2) kodiert. Außerdem teilen sich die Textaufgaben des instruktiven Faktors eine (teil-)spezifische häufige Kodierung mit dem komprimierenden Faktor. Die Textaufgaben beider Faktoren wurden häufig mit keiner Zuordnung zu einem Transferbezug für die Hauptkategorie Transferbezug (gelb) kodiert. Zur Interpretation wird besonders der Vergleich mit den genannten Faktoren hergestellt. 
5. Textaufgaben des instruktiven Faktors: Die Textaufgaben des instruktiven Faktors haben wie die des beschreibenden Faktors häufig eine rechnerische Argumentation. Unterschiede ergeben sich zwischen dem instruktiven und dem beschreibenden Faktor in der Hauptkategorie Interaktion. Instruktive Textaufgaben zeichnen sich insbesondere durch eine unpersönliche Interaktion, mit dem Fokus auf den Inhalt aus. Außerdem unterscheiden sich die Textaufgaben beider Faktoren in der Hauptkategorie Transferbezug, die für Textaufgaben des instruktiven Faktors häufig keine Zuordnung erhält. Auch die Textaufgaben des komprimierenden Faktors erhalten häufig keine Zuordnung. Die Textaufgaben des instruktiven Faktors unterscheiden sich jedoch von denen des komprimierenden Faktors in der Häufigkeit der Kodierung in den Hauptkategorien Darstellungen und Tätigkeit. Aufgaben des instruktiven Faktors haben oftmals keine Darstellungen und es wird außermathematisch modelliert (vgl. Abbildung 9.9). Die rechnerische, jedoch unpersönliche Interaktion weist auf die Bedeutung der inhaltlichen Vermittlung hin. Der Fokus liegt jedoch nicht auf Darstellungen und dem Umgang mit diesen, sondern auf über den Text formulierten außermathematischen Modellierungen, deren Transferbezug nicht eindeutig ist. Diese fehlende Eindeutigkeit des Transferbezugs in einer außermathematischen Modellierung spricht für eine inhaltsorientierte Passung zwischen Mathematik und Realität. Der außermathematische Bezug dient als Übersetzungsgrundlage für den fachlichen Inhalt, ohne dass Ersterer eine tragende Relevanz für die Aufgabenlösung hat. Darauf deutet auch die häufig rechnerische Argumentation hin. Anhand der Interpretationen wird die Gruppe der Textaufgaben des instruktiven Faktors mit der Bezeichnung des Aufgabentyps fachliche Aufgaben charakterisiert (vgl. Tabelle 9.3).

In Tabelle 9.3 ist die Zusammenfassung der Bezeichnungen der Textaufgaben je Faktor nach der Interpretation dargestellt.

Es wird deutlich, dass die Textaufgaben jedes Faktors eine AufgabentypenBezeichnung erhalten. Typischerweise sind die Textaufgaben des erklärenden Faktors sequenzielle Aufgaben, die des komprimierenden Faktors ikonische Aufgaben, die des beschreibenden Faktors rechnerische Aufgaben, die des informativen Faktors sachliche Aufgaben und die des instruktiven Faktors fachliche Aufgaben.

Für die unterschiedlichen Aufgabentypen ergeben sich typische Ähnlichkeiten und Unterschiede, die in Abbildung 9.10 je Faktor zusammengefasst sind. Die Abbildung verdeutlicht diese Ähnlichkeiten und Unterschiede durch ähnliche Musterbildung in dem dargestellten Koordinatendiagramm. 
Tabelle 9.3 Zusammenfassung der Bezeichnungen eines Aufgabentyps für die ausgewählten Textaufgaben der jeweiligen Faktoren

\begin{tabular}{l|l|l}
\hline Faktor & Aufgabentyp & Definition \\
\hline Erklärend & Sequenzielle Aufgaben & $\begin{array}{l}\text { Unter sequenziellen Aufgaben können } \\
\text { Textaufgaben im Mathematikunterricht } \\
\text { verstanden werden, die sich besonders } \\
\text { durch eine strukturierte Unterteilung der } \\
\text { Aufgabenelemente auszeichnen. }\end{array}$ \\
\hline Komprimierend & Ikonische Aufgaben & $\begin{array}{l}\text { Unter ikonischen Aufgaben können } \\
\text { Textaufgaben im Mathematikunterricht } \\
\text { verstanden werden, die sich besonders } \\
\text { durch die Verwendung von mathematischen } \\
\text { Darstellungen auszeichnen. }\end{array}$ \\
\hline Beschreibend & Rechnerische Aufgaben & $\begin{array}{l}\text { Unter rechnerischen Aufgaben können } \\
\text { Textaufgaben im Mathematikunterricht } \\
\text { verstanden werden, die auf die Berechnung } \\
\text { fokussieren. }\end{array}$ \\
\hline Informativ & Sachliche Aufgaben & $\begin{array}{l}\text { Unter sachlichen Aufgaben können } \\
\text { Textaufgaben im Mathematikunterricht } \\
\text { verstanden werden, bei denen } \\
\text { lösungsrelevanten Sachinhalt vorkommen. }\end{array}$ \\
\hline Instruktiv & Fachliche Aufgaben & $\begin{array}{l}\text { Unter fachlichen Aufgaben können } \\
\text { Textaufgaben im Mathematikunterricht } \\
\text { verstanden werden, mit einer } \\
\text { inhaltsorientierten Passung. }\end{array}$ \\
\hline & &
\end{tabular}

Bei einer Betrachtung von beispielsweise den Hauptkategorien Interaktion (dunkelgrün) und Tätigkeit (hellgrün), zeigt sich, dass die Textaufgaben der Faktoren erklärend, informativ und instruktiv häufig die Kodierung der unpersönlichen Interaktion (2) und der außermathematischen Modellierung (2) gemeinsam aufweisen. Ebenfalls ist die ähnlich häufige Kodierung zwischen den Textaufgaben des beschreibenden und informativen Faktors erkennbar, die sich nur in der häufigen Kodierung der Hauptkategorie Interaktion unterscheiden.

Die Abbildung 9.10 kann zum Vergleich der typischen Ähnlichkeiten und Unterschiede dienen. Exemplarisch kann ein möglicher Vergleich der Textaufgaben des erklärenden Faktors mit den anderen Textaufgaben demonstriert werden. So ähneln sich beispielsweise Textaufgaben des erklärenden Faktors (sequenzielle Aufgaben) und Textaufgaben des komprimierenden Faktors (ikonische Aufgaben) in der Häufigkeit der Kodierung der Subkategorien in den Hauptkategorien mathematische Argumentation, Darstellungen und Interaktion und unterscheiden 


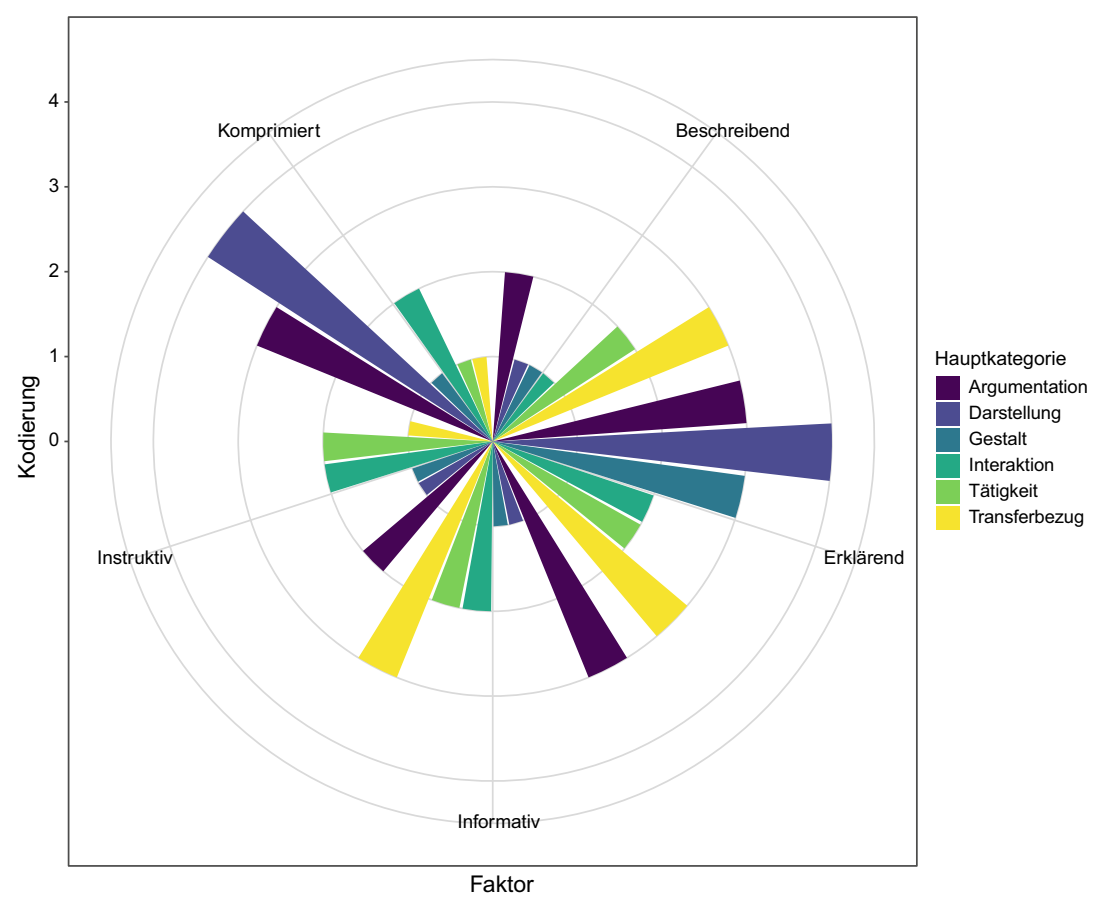

Abbildung 9.10 Zusammenfassung der Darstellungen der häufigen Kodierung der Hauptkategorien der Textaufgaben für alle Faktoren (Eigene Erstellung)

sich in Gestalt und mathematischer Tätigkeit. Textaufgaben des erklärenden Faktors (sequenzielle Aufgaben) und solche des beschreibenden Faktors (rechnerische Aufgaben) erhalten häufig eine Kodierung der Subkategorien in den Hauptkategorien mathematische Tätigkeit und Transferbezug. Die Textaufgaben unterscheiden sich in der Kodierung der Häufigkeit der Subkategorien der Hauptkategorien Gestalt, Interaktion, Argumentation und Darstellung.

Für Textaufgaben des erklärenden Faktors (sequenzielle Aufgaben) und solche des informativen Faktors (sachliche Aufgaben) ergeben sich Unterschiede in der Häufigkeit der Kodierung der Subkategorien der Hauptkategorien Darstellungen, Gestalt und Ähnlichkeiten in Bezug auf Argumentation, Tätigkeit, Transferbezug und Interaktion. In Anbetracht der Textaufgaben des erklärenden Faktors (sequenzielle Aufgaben) und der des instruktiven Faktors (fachliche Aufgaben) zeigen sich Unterschiede in Bezug auf die Häufigkeit der Kodierung der Subkategorien 
der Hauptkategorien Argumentation, Darstellungen, Transferbezug, Gestalt und Ähnlichkeiten in den Hauptkategorien Tätigkeit und Interaktion.

Resümee: Durch die qualitative Vertiefungsanalyse konnte das in Abschnitt 6.1 formulierte vierte Ziel der Konzeptualisierung eines Instruments zur sprachlichen Variation von mathematischen Textaufgaben erreicht werden. Ziel war es, inhaltsund kontextbezogene Merkmale der Aufgaben mit den sprachlichen Faktoren in Beziehung zu bringen. Der Grund dieses Ziels war es, die aus der Theorie in Kapitel 3 und Kapitel 4 hergeleitete Relevanz der Beziehung von sprachlichen und kontextuellen Veränderungen in das Instrument einzubeziehen. Die qualitative Inhaltsanalyse, insbesondere die Interpretation sowie Tabelle 9.3, macht deutlich, dass sich die sprachlichen Faktoren und die inhaltlichen und kontextuellen Merkmale auf empirischer Basis miteinander in Beziehung bringen lassen. Diese Beziehung zeichnet sich durch das gemeinsame Vorkommen von sprachlichen Merkmalen aus. Die Beziehung führt zu einer Häufung der Verwendung charakteristischer fachlicher und kontextueller Merkmale und den theoretischen Zusammenhang von sprachlicher und kontextueller Veränderung auf der Ebene von mathematischen Textaufgaben bestätigt.

\subsection{Zusammenfassung}

Bei der zweiten qualitativen Vertiefungsanalyse wurde die Systematisierung von Textmerkmalen aus der ersten quantitativen Studie (vgl. Kapitel 7) zur Feststellung von charakteristischen Aufgaben durch die Ausprägungen der Faktorenwerte verwendet. Anhand der Selektion von spezifischen Beispielen für die Faktoren wurden $N=49$ Textaufgaben aus dem Gesamtdatensatz für die qualitative Vertiefungsanalyse genutzt.

Auf Grundlage der ausgewählten Textaufgaben wurde durch ein deduktivinduktives Vorgehen ein Kategoriensystem entwickelt. Mithilfe dieses Systems, das den Gütekriterien einer qualitativen Analyse entspricht, wurden die charakteristischen Aufgaben kodiert.

Bereits durch die fallspezifische Analyse wurde deutlich, dass Ähnlichkeiten der Textaufgaben innerhalb des Faktors und Unterschiede der Textaufgaben zwischen den Faktoren in Bezug auf fachliche und kontextuelle Merkmale bestehen.

Die Ergebnisse und die Darstellung der Kodierung, insbesondere der häufigsten Kodierung, stellen Spezifika der unterschiedlichen Textaufgaben je Faktor dar und 
bieten die Möglichkeit, die Textaufgaben zu typisieren. Die Ergebnisse der Typisierung zeigen, dass die Textaufgaben des erklärenden Faktors (sprachlich) mit sequenziellen Aufgaben (fachlich-kontextuell), die des komprimierenden Faktors (sprachlich) mit ikonischen Aufgaben (fachlich-kontextuell), die des beschreibenden Faktors (sprachlich) mit rechnerischen Aufgaben (fachlich-kontextuell), die des informativen Faktors (sprachlich) mit sachlichen Aufgaben (fachlichkontextuell) und die des instruktiven Faktors (sprachlich) mit fachlichen Aufgaben (fachlich-kontextuell) in Beziehung stehen.

Durch die empirisch dargestellte Verbindung sowohl der sprachlichen Faktoren als auch der fachlichen und kontextuellen Kategorien indizieren die Ergebnisse der qualitativen Vertiefungsanalyse damit, dass sich die theoretisch definierte Beziehung zwischen Sprache und Kontext (vgl. u. a. Kapitel 3 und Kapitel 4) empirisch darstellen lässt und in der Konzeptualisierung eines Instruments zur sprachlichen Variation einbezogen werden kann.

Ausblick: Die drei empirischen Anteile dieser Arbeit erfüllen die in Abschnitt 6.1 formulierten Zielvoraussetzungen für das empirisch-induktive Instrument zur sprachlichen Variation von mathematischen Textaufgaben. Um die Resultate in einen stärkeren Zusammenhang zu bringen, werden im anschließenden Kapitel 10 alle Ergebnisse in Beziehung gebracht.

Open Access Dieses Kapitel wird unter der Creative Commons Namensnennung 4.0 International Lizenz (http://creativecommons.org/licenses/by/4.0/deed.de) veröffentlicht, welche die Nutzung, Vervielfältigung, Bearbeitung, Verbreitung und Wiedergabe in jeglichem Medium und Format erlaubt, sofern Sie den/die ursprünglichen Autor(en) und die Quelle ordnungsgemäß nennen, einen Link zur Creative Commons Lizenz beifügen und angeben, ob Änderungen vorgenommen wurden.

Die in diesem Kapitel enthaltenen Bilder und sonstiges Drittmaterial unterliegen ebenfalls der genannten Creative Commons Lizenz, sofern sich aus der Abbildungslegende nichts anderes ergibt. Sofern das betreffende Material nicht unter der genannten Creative Commons Lizenz steht und die betreffende Handlung nicht nach gesetzlichen Vorschriften erlaubt ist, ist für die oben aufgeführten Weiterverwendungen des Materials die Einwilligung des jeweiligen Rechteinhabers einzuholen.

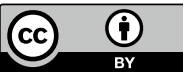

